This is the pre-peer reviewed version of the following article:

Perumbilavil S., López-Ortega A., Tiwari G.K., Nogués J., Endo T., Philip R.. Enhanced Ultrafast Nonlinear Optical Response in Ferrite Core/Shell Nanostructures with Excellent Optical Limiting Performance. Small, (2018). 14. 1701001: - . 10.1002/smll.201701001,

which has been published in final form at https://dx.doi.org/10.1002/smll.201701001. This article may be used for non-commercial purposes in accordance with Wiley Terms and Conditions for Use of Self-Archived Versions. 


\section{Enhanced Ultrafast Nonlinear Optical Response in Ferrite Core/Shell Nanostructures with Excellent Optical Limiting Performance}

Sreekanth Perumbilavil, Alberto López-Ortega*, Gaurav Kumar Tiwari, Josep Nogués, Tamio Endo, Reji Philip

Mr. Sreekanth Perumbilavil

Ultrafast and Nonlinear Optics Lab, Light and Matter Physics Group, Raman Research Institute, Bangalore 560080, India.

Present address: Photonics Laboratory, Tampere University of Technology, P.O.Box 692, FI33101, Tampere, Finland.

Dr. Alberto López-Ortega

CIC nanoGUNE, Tolosa Hiribidea, 76 E-20018 Donostia-San Sebastian, Spain.

E-mail: lopezortega.alberto@gmail.com

Mr. Gaurav Kumar Tiwari

Ultrafast and Nonlinear Optics Lab, Light and Matter Physics Group, Raman Research Institute, Bangalore 560080, India.

Prof. Josep Nogués

Catalan Institute of Nanoscience and Nanotechnology (ICN2), CSIC and The Barcelona Institute of Science and Technology, Campus UAB, Bellaterra, 08193 Barcelona, Spain. Institució Catalana de Recerca i Estudis Avançats (ICREA), Barcelona, Spain.

Prof. Tamio Endo

Emeritus Professor, Mie University, 514-8507, Mie, Japan

Faculty of Engineering, Gifu University, 501-1193 Gifu, Japan.

Prof. Reji Philip

Ultrafast and Nonlinear Optics Lab, Light and Matter Physics Group, Raman Research Institute, Bangalore 560080, India.

Keywords: nonlinear absorption; core/shell nanoparticles; optical limiting; oxide heterostructures

Nonlinear optical nanostructured materials are gaining increased interest as optical limiters for various applications, although many of them may suffer from reduced efficiencies at highlight fluences due to photo-induced deterioration. Here we report the nonlinear optical properties of ferrite core/shell nanoparticles showing their robustness for ultrafast optical limiting applications. When excited by $100 \mathrm{fs}$ ultrashort laser pulses the effective two-photon absorption (2PA) coefficient shows a non-monotonic dependence on the shell thickness, with 
a maximum value obtained for thin shells. In view of the local electric field confinement, this indicates that core/shell may be an advantageous morphology to improve the nonlinear optical parameters. These nanoparticles exhibit excellent optical limiting performance with effective 2PA coefficients in the range of $10^{-12} \mathrm{~cm} / \mathrm{W}$ for a $100 \mathrm{fs}$ excitation, and optical limiting threshold fluences in the range of $1.7 \mathrm{~J} / \mathrm{cm}^{2}$. These values are comparable to, or better than, most of the recently reported optical limiting materials. ${ }^{[1-10]}$ The quality of the open aperture Z-scan data recorded from repeat measurements at intensities as high as $35 \mathrm{TW} / \mathrm{cm}^{2}$, indicate their considerably high optical damage thresholds in a toluene dispersion, ensuring their robustness in practical applications. Thus, the high photostability combined with the remarkable nonlinear optical properties make these ferrite nanoparticles excellent candidates for ultrafast optical limiting applications.

\section{Introduction}

Nanostructured materials are gaining increased interest both in industry and research due to their novel and enhanced properties with respect to their bulk counterparts, which are leading to countless applications in very diverse fields. ${ }^{[11-13]}$ In particular, in the field of optics, nanoparticles are finding widespread uses. ${ }^{[14,15]}$ For example, (i) metallic nanoparticles have plasmonic properties which depend strongly on their size and shape, the dielectric environment and the inter-particle distance; ${ }^{[16]}$ (ii) transparent polymer films embedded with nanoparticles have been shown to have tunable ultrahigh refractive indexes; ${ }^{[17]}$ (iii) arrays of metallic nanoparticles behave as photonic crystals; ${ }^{[18]}$ and (iv) nanostructures can present large third-order optical nonlinearities, with applications in fields such as optical telecommunication, optical data storage, optical computing and information processing. ${ }^{[19]}$

Many nonlinear optical (NLO) materials have been explored for optical limiting applications. Optical limiters are devices used for protecting light detectors or other optical components 
(including human eyes) from accidental exposure to harmful high intensity radiation. Ideal optical limiters should be fully transparent to light of low and moderate intensity, but fully opaque to high intensity light. Despite their importance, the capabilities of existing optical limiters are still far from ideal. Thus, diverse approaches, using different types of materials such as metallic, semiconductor or organic,,$^{[1,20,21]}$ are being investigated to improve their performance. However, high intensities result in photo-fragmentation and ligand desorption in most of these materials, resulting in irreversible material damage. ${ }^{[2,22,23]}$ Thus, exploring new materials with high stability (with higher intensity thresholds for laser-induced thermal damage and dielectric breakdown) suitable for use in harsh/reactive environments is crucial for the development of practical devices for optical limiting applications.

Interestingly, magnetic spinel ferrites $\left(\mathrm{M}_{\mathrm{x}} \mathrm{Fe}_{3-\mathrm{x}} \mathrm{O}_{4}\right.$, where $\mathrm{M}=$ transition metal, e.g., $\mathrm{Fe}, \mathrm{Co}$, $\mathrm{Mn}, \mathrm{Ni}$, or $\mathrm{Zn}$ ) are versatile nanomaterials which are known to be highly stable even in extreme environments. ${ }^{[24]}$ However, compared to other materials, optical nonlinearities in ferrites have been relatively unexplored. ${ }^{[25-27]}$ Although modifications in optical nonlinearity caused by the inclusion of different transition metals into a spinel ferrite system have been reported, ${ }^{[28]}$ many archetypical ferrite materials like $\mathrm{MnFe}_{2} \mathrm{O}_{4}$ have not yet been investigated.

Core/shell architectures have become increasingly appealing among nanomaterials to develop efficient ways to functionalize and improve the properties of single-phase nanoparticles ${ }^{[12,29-}$ ${ }^{32]}$ in distinct fields such as microelectronics, biomedicine, catalysis, optics, and magnetism, among many others. ${ }^{[33-43]}$ In the particular case of optical limiters, core/shell structures have been used to make certain materials stable at extreme conditions, where chemically inert oxide shells have been grown for protective purposes. ${ }^{[44]}$ However, the use of non-optimized protective shells may weaken the optical limiting capabilities of the material. On the other hand, metal-semiconductor core/shell composite nanoparticles have been theoretically predicted to exhibit strong enhancement in the nonlinear response compared to their single 
components counterparts. ${ }^{[45]}$ Nevertheless, investigations on core/shell structures with optically active shells for optical limiting purposes are still rather scarce, ${ }^{[46]}$ in particular for highly stable materials like all-oxide structures.

Here we report ultrafast optical nonlinearities measured in a series of ferrite core/shell nanoparticles, $\mathrm{MnFe}_{2} \mathrm{O}_{4} / \mathrm{CoFe}_{2} \mathrm{O}_{4}$ and $\mathrm{CoFe}_{2} \mathrm{O}_{4} / \mathrm{MnFe}_{2} \mathrm{O}_{4}$, with different core diameter and shell thickness, studied by the open aperture Z-scan technique. The results show that for 100 fs, $800 \mathrm{~nm}$ laser pulses, the core/shell architecture leads to an enhanced optical limiting performance, with optical limiting efficiencies comparable to, or better than, those of benchmark materials reported in the literature. ${ }^{[1-10]}$ It may be noted that the $800 \mathrm{~nm}$ wavelength is rather common for biomedical applications given the minimal absorption of water (and other biological fluids) around this wavelength. ${ }^{[47]}$

\section{Results and discussion}

Three different series of $\mathrm{CoFe}_{2} \mathrm{O}_{4} / \mathrm{MnFe}_{2} \mathrm{O}_{4}$ core/shell nanoparticles have been synthesized; two based on Co-ferrite cores and Mn-ferrite shells (Co1@Mn and Co2@Mn, with two different core sizes) and one in the inverted $\mathrm{Mn}_{2} \mathrm{Fe}_{2} \mathrm{O}_{4} / \mathrm{CoFe}_{2} \mathrm{O}_{4}(\mathrm{Mn} 1 @ \mathrm{Co}$ ) core/shell architecture. Figures 1 and S1 show the transmission electron microscopy (TEM) images and their respective particle size histograms for the three series: Mn1@Co, Co1@Mn and Co2@Mn. Single phase seeds for the Mn1@Co and Co1@Mn series (i.e., Co1 and Mn1) present similar 6(1) nm particle sizes. After the different shell deposition steps both core/shell series show the similar increase in overall diameter, reaching particle sizes of 9,11 and 12 nm, i.e., shell thicknesses of 1.5, 2.5 and $3 \mathrm{~nm}$, respectively (see Table S1). Conversely, the Co2@Mn series starts with slightly larger $8(1)$ nm $\mathrm{CoFe}_{2} \mathrm{O}_{4}$ seeds and similar shell thickness increase, i.e., 0.5, 1.5 and $2 \mathrm{~nm}$, after each deposition step (see Table S1). All nanoparticles depict a rather regular spherical shape with a narrow particle size distribution $(<15 \%)$. In 
order to corroborate the core/shell structure of the nanoparticles an electron energy loss spectroscopy (EELS) analysis of the structure has been performed. ${ }^{[48,49]}$ The EELS mappings for the sample Co1@Mn1 clearly confirm the core/shell morphology, where manganese and cobalt ions are confined only in the shell and core regions, respectively (see Figure 2). The xray diffraction (XRD) patterns for the Co1 series, depicted in the Figure S2, show the formation of a single crystallographic phase, which can be indexed as cubic spinel. The series of diffractograms reveals the expected gradual narrowing of the peaks associated with the increase of particle size. The crystallite sizes, evaluated from the diffraction patterns, are consistent with those obtained from the TEM images, indicating the growth of single crystalline nanoparticles (see Table S1). In addition, the cell parameter depicts a linear increase as the shell thickness becomes larger, in agreement with the expected variation of the unit cell values between the $\mathrm{CoFe}_{2} \mathrm{O}_{4}$ core and the $\mathrm{MnFe}_{2} \mathrm{O}_{4}$ shell, (i.e. a $\mathrm{CoFe}_{2} \mathrm{O} \approx \approx .4$ and $\mathrm{a}_{\mathrm{MnFe} 2 \mathrm{O} 4} \approx 8.5 ;$ see Table $\left.\mathrm{S} 1\right)$. Interestingly, the core/shell structure is further supported by their magnetic behaviour (see Supplementary Information and Figure S3). Moreover, the linear absorption of the ferrite core/shell nanoparticles in the visible-near infrared region has been measured showing the typical profile for iron-based oxides with a decay of the absorption from lower to higher wavelengths (see Figure S4). ${ }^{[50]}$

The nonlinear optical response of the ferrite core/shell nanoparticles dispersed in toluene was measured at $800 \mathrm{~nm}$ by the open aperture Z-scan technique, using ultrashort laser pulses of 100 fs duration. Note that the calibration curves, i.e., Z-scans measured in pure toluene, do not show any nonlinear absorption (see Figure S5).

The normalized transmittance of the sample (i.e., the measured transmission normalized to the linear transmission at low input light levels) plotted as a function of the input optical intensity and fluence is given in Figure 3 (Co1@Mn series), Figure S6 (Mn1@Co series) and Figure S7 (Co2@Mn series), respectively. These curves are calculated from the open aperture Z-scan 
curves (which are shown as the insets) using the divergence properties of a focusing Gaussian beam (see the Experimental Section). For all three series of nanoparticles the normalized transmittance is strongly dependent on the input intensity, depicting a strong absorptive nonlinearity. The smooth valley in the Z-scans (which is symmetric about the beam focus, $\mathrm{z}=$ 0) confirms a strong reduction in the optical transmission at higher intensities. A weaker, but measurable increase in transmission can be seen at moderate intensities, which arises from saturable absorption.

Depending on the linear absorption spectrum of the sample, excitation wavelength, laser fluence, intensity and pulse width, nonlinear absorption can originate from diverse processes such as multi-photon absorption (two-photon absorption (2PA), three-photon absorption (3PA) etc.), excited state absorption (ESA), and saturable absorption (SA). ${ }^{[51,52]}$ Since the samples are concentrated enough to have some linear absorption at $800 \mathrm{~nm}$ (the linear transmission of $70 \%$ indicates about $22 \%$ linear absorption, after accounting for about $8 \%$ reflection losses from the sample cuvette), ESA is a logical contributor to the observed nonlinear absorption. ${ }^{[52,53]}$ At the same time, given the high intensities provided by the ultrashort laser pulses (beyond $10^{13} \mathrm{~W} / \mathrm{cm}^{2}$ in the present study) $2 \mathrm{PA}$ will play also a significant role. ${ }^{[52,53]}$ Therefore we refer to the combined effect as effective 2PA, following convention. ${ }^{[52]}$ Taking the SA seen at moderate intensities also into consideration, the nonlinear absorption coefficient can be written as ${ }^{[54]}$

$$
\alpha(z)=\frac{\alpha_{0}}{1+\left(I / I_{s}\right)}+\beta I
$$

where $\beta$ is the effective 2PA coefficient and $I_{s}$ is the saturation intensity. $\alpha_{0}$ is the linear absorption coefficient, and I is the incident intensity. The corresponding light propagation equation is given by: 


$$
\frac{d I}{d z^{\prime}}=-\left[\frac{\alpha_{0}}{1+\left(I / I_{s}\right)}+\beta I\right] I
$$

where $\mathrm{z}^{\prime}$ denotes the axis of propagation through the sample. The solution to this equation is numerically fitted to the measured nonlinear transmission data to obtain the values of $I_{s}$ and $\beta$, which are given in Table S2.

From Figure 4 it can be seen that $\beta$ initially increases with shell thickness for both Co-ferrite and $\mathrm{Mn}$-ferrite morphologies. Interestingly, $\beta$ turns out to be maximum for particles of intermediate sizes, for which the shells are relatively thin. The enhancement is in the range of $22 \%$ to $50 \%$ when compared to the pure ferrite cores. When the particle size increases beyond the intermediate range due to thicker shells, however, $\beta$ is found to decrease. The nonlinearity improvement observed for nanoparticles with thin shells may be attributed to the local electric field enhancement associated with the core/shell structure. Considering the optical irradiation of core/shell composite particles dispersed in a surrounding dielectric medium, the local field enhancement can be calculated by solving Laplace's equation with appropriate boundary conditions, employing FDTD (finite-difference time-domain) simulation techniques. Note that, the local field enhancement will be most prominent in metal nanoparticles which have a surface plasmon resonance, where it will be maximized when the optical field is in exact resonance with the surface plasmon frequency. ${ }^{[45]}$ However, in oxide nanoparticles (typically insulating) surface plasmon resonances do not occur. For the core/shell/dielectric morphology, the FDTD simulations show that the local field enhancement is a minimum within the shell volume, compared to the other regions (see Fig.6 and the corresponding discussion below). The increase of the shell volume for a fixed core volume obviously reduces the core volume fraction in the case of thicker shells. Since the core has a higher local field enhancement compared to the shell, this leads to an overall reduction in the nonlinearity 
of the nanoparticles with thicker shells. Note that, similar non-monotonic size dependent enhancement of ultrafast optical nonlinearity (fs or ps pulse excitations) has been previously reported in CdSe/CdS colloidal core/shell nanocrystals, ${ }^{[55]}$ Mn-doped ZnSe nanocrystals ${ }^{[56]}$ and $\mathrm{Au}-\mathrm{SiO}_{2}$ core/shell nanoparticles. ${ }^{[57]}$ However, in contrast to quantum dots and noble metals, ferrite nanoparticles are environmentally friendly, inexpensive and very stable with temperature or in reactive environments. Additionally, oxide nanoparticles do not present any bleaching effects, which could be an important limitation for the application of quantum dots in devices.

In order to better understand the improved nonlinear optical response due to the field enhancement we selected the Co1 and Mn1 series for further theoretical analysis (however, note that samples from all three series exhibit the same trend of nonlinearity). From the $\beta$ values obtained for the seed nanoparticles of the Co1 and Mn1 series, the corresponding thirdorder nonlinear susceptibility values of the core materials, $\chi_{\mathrm{m}}{ }^{(3)}$, can be determined by the expression: $:^{[54]}$

$$
\chi_{\mathrm{m}}^{(3)}=\beta \lambda \mathrm{cn}_{0}^{2} \varepsilon_{0} / 3 \pi
$$

where $\lambda$ is excitation wavelength, $\mathrm{n}_{0}$ is the linear refractive index, $\mathrm{c}$ is the speed of light in vacuum and $\varepsilon_{0}$ is the dielectric constant. For Co1 seed and Mn1 seed, $\chi_{\mathrm{m}}{ }^{(3)}$ is calculated to be $2.4 \times 10^{-21} \mathrm{~m}^{2} / \mathrm{V}^{2}$ and $1.96 \times 10^{-21} \mathrm{~m}^{2} / \mathrm{V}^{2}$, respectively. Now, according to the local field theory, the effective nonlinear susceptibility of the core/shell system dispersed in a dielectric medium (toluene in the present case) is given by ${ }^{[55,58]}$.

$$
\chi_{\text {eff }}^{(3)}=\mathrm{P}\left(3 \varepsilon_{0} /\left(2 \varepsilon_{0}+\varepsilon_{\text {eff }}\right)\right)^{4} \chi_{\mathrm{m}}^{(3)}
$$

where $\mathrm{P}$ is the volume fraction of nanoclusters in the dispersion, and $\left(3 \varepsilon_{0} /\left(2 \varepsilon_{0}+\varepsilon_{\text {eff }}\right)\right)^{4}$ is the local field enhancement factor. $\varepsilon_{\text {eff }}$ is given by $\varepsilon_{0}+3 \varepsilon_{0} \mathrm{~Pb}$, where $\mathrm{b}=\left(\varepsilon_{2}-\right.$ $\left.\varepsilon_{0}+\left(\varepsilon_{0}+2 \varepsilon_{2}\right) \rho_{1} \mathrm{f}^{3}\right) /\left(\varepsilon_{2}+2 \varepsilon_{0}+2\left(\varepsilon_{2}-\varepsilon_{0}\right) \rho_{1} \mathrm{f}^{3}\right)$, with $\rho_{1}=\left(\varepsilon_{1}-\varepsilon_{2}\right) /\left(\varepsilon_{1}+2 \varepsilon_{2}\right) ; \mathrm{f}$ is the ratio between the radii of core $\left(\mathrm{r}_{1}\right)$ and core/shell $\left(\mathrm{r}_{2}\right)$; and $\varepsilon_{0}, \varepsilon_{1}$ and $\varepsilon_{2}$ are the dielectric constants of the dielectric, 
core material and the shell material, respectively. For the Co1 series, $\varepsilon_{0}, \varepsilon_{1}$ and $\varepsilon_{2}$ are 2.21 , 5.29 and 6.15 respectively, while for the Mn1 series, these values are 2.21, 6.15 and 5.29 respectively.

The theoretically calculated (using Equation 4) and experimentally estimated $\chi_{\text {eff }}{ }^{(3)}$ values, plotted as a function of shell thickness (Figure 5), are in excellent agreement with each other. The nonlinear susceptibility increases up to a shell thickness of $2 \mathrm{~nm}$, and slightly decreases thereafter. The measured values show a slight deviation from the theoretical curve for samples with thicker shells, which might be due to the presence of non-radiative surface traps formed due to the strain and lattice mismatch at the core/shell interface. ${ }^{[55,59,60]}$

To calculate the distribution of enhanced electric fields in and around the core/shell nanoparticles dispersed in toluene, FDTD simulations were carried out. In the FDTD method space is divided into discrete grids and then the electromagnetic field is evolved in time by using discrete steps. The electric field vector components in a volume of space are solved at a given instant in time, and the magnetic field vector components in the same spatial volume are solved at the next instant in time. The process is repeated until the desired transient or steady-state electromagnetic field behavior is fully evolved. For our FDTD simulations, optical constants were obtained from literature for $\mathrm{CoFe}_{2} \mathrm{O}_{4}{ }^{[61]}$ and $\mathrm{MnFe}_{2} \mathrm{O}_{4}{ }^{[62]}$ The thinnest shell $(1.5 \mathrm{~nm})$ enhances the field by about $11 \%$ compared to the bare seeds, while the $2.5 \mathrm{~nm}$ shell enhances the field by about $100 \%$ (see Figure 6). No further enhancement is seen for the $3 \mathrm{~nm}$ shell. This trend is in qualitative agreement with the enhancement of $\beta$ and $\chi_{\text {eff }}^{(3)}$ values measured for the nanoparticles in the presence of thin shells. FDTD simulations carried out for excitation at the wavelength of $400 \mathrm{~nm}$ (where the particles show a much stronger absorption) are given in Figure S8. Since the strong absorption does not arise from SPR, no relevant enhancement in the field is observed. In fact, for the Co1Mn2 nanoparticle, the enhancement at $800 \mathrm{~nm}$ is found to be larger than that at $400 \mathrm{~nm}$. 
Remarkably, the experimental $\beta$ values are in the range of $10^{-12}$ to $10^{-13} \mathrm{~cm} / \mathrm{W}$, which are comparable to those recently reported in $\mathrm{CuO} / \mathrm{Graphene}$ hybrids $\left(\beta \sim 10^{-12} \mathrm{~cm} / \mathrm{W}\right)$, ${ }^{[3]}$ reduced graphene oxide $\left(\beta \sim 10^{-12} \mathrm{~cm} / \mathrm{W}\right),{ }^{[1]} \mathrm{BiFeO}_{3}\left(\beta \sim 10^{-12} \mathrm{~cm} / \mathrm{W}\right),{ }^{[4]} \mathrm{Fe}_{2} \mathrm{O}_{3}\left(\beta \sim 10^{-13} \mathrm{~cm} / \mathrm{W}\right),{ }^{[5]}$ $\mathrm{SiO}_{2}-\mathrm{Se}\left(\beta \sim 4.8 \times 10^{-13} \mathrm{~cm} / \mathrm{W}\right),{ }^{[6]} \mathrm{CuS}$ nanoparticles $\left(\beta \sim 6 \times 10^{-13} \mathrm{~cm} / \mathrm{W}\right)$ and $\mathrm{CuS}$ quantum dots $\left(\beta \sim 2.3 \times 10^{-12} \mathrm{~cm} / \mathrm{W}\right) .^{[7]}$ The $\beta$ values are also comparable to those of CdSe quantum dots (in the size range of 2 to $3.9 \mathrm{~nm}$ ) dispersed in hexane, which are in the range of $10^{-12}$ $\mathrm{cm} / \mathrm{W} \cdot{ }^{[8]}$ In addition, the optical limiting efficiency of the ferrite core/shell nanoparticles is very good, with limiting threshold (input fluence at which the transmission drops to $50 \%$ of the linear transmission value) values in the range 1.66 to $2.24 \mathrm{~J} / \mathrm{cm}^{2}$ (see Table S2). These are similar to or better than those of previously reported bench-mark materials like $\mathrm{C}_{60}\left(2 \mathrm{~J} / \mathrm{cm}^{2}\right)$, carbon black $\left(2.2 \mathrm{~J} / \mathrm{cm}^{2}\right),{ }^{[2]}$ graphene-Ag $\left(1.9 \mathrm{~J} / \mathrm{cm}^{2}\right),{ }^{[9]}$ and graphene-Pt $\left(1.8 \mathrm{~J} / \mathrm{cm}^{2}\right),{ }^{[10]}$ and are considerably better compared to those obtained for Pt $\left(33.1 \mathrm{~J} / \mathrm{cm}^{2}\right)$ and Pd $\left(24.2 \mathrm{~J} / \mathrm{cm}^{2}\right)$ nanoparticles. ${ }^{[10]}$

Finally, to evaluate the optical damage behaviour of the ferrite core/shell particles, samples Co1 and Co1@Mn1 were irradiated using high intensity laser pulses ${ }^{[63,64]}$ (fluence is 3.5 $\mathrm{J} / \mathrm{cm}^{2}$, beam spot size at focus is $24 \mu \mathrm{m}$, and intensity is $35 \mathrm{TW} / \mathrm{cm}^{2}$ ). The Z-scan curves do not exhibit signs of laser induced damage, like loss of symmetry of the curve, or scattering of data points near the beam focus $(\mathrm{z}=0)$, even after several runs of the experiment. This indicates that when the nanoparticles are measured in solution, sample damage is not observed at least up to $35 \mathrm{TW} / \mathrm{cm}^{2}$. It may be noted that high intensities can be achieved at relatively low pulse energies, provided that the pulse width is small, as in the present case. Since lower energies result in lesser heating, thermal damage will be less. On the other hand high optical intensities can result in dielectric breakdown of the material, but since the Z-scan curves remain robust even after repeated measurements, we can assume that dielectric 
breakdown does not occur in these samples. To assess whether the nanoparticles are indeed getting photodamaged at the beam focus is rather complex. However, even if that was the case, the diffusion of the nanoparticles in the liquid medium would ensure that the irradiated volume is constantly being restored, so that the optical limiting performance is not compromised (thus, such high optical stability might not be attained if the particles are dispersed in a solid matrix). The intensity of $35 \mathrm{TW} / \mathrm{cm}^{2}$ is well above the damage thresholds of $\mathrm{Au}$ nanowires $\left(3 \mathrm{TW} / \mathrm{cm}^{2}\right)^{[65]}$ and multi-walled carbon nanotubes $\left(11 \mathrm{TW} / \mathrm{cm}^{2}\right)$, and comparable to multilayered graphene embedded in proteins $\left(34 \mathrm{TW} / \mathrm{cm}^{2}\right) .{ }^{[66]}$ Consequently, their remarkably high endurance of intense ultrashort laser pulses ensures that these ferrite core/shell nanoparticles are excellent materials for optical limiting applications.

\section{Conclusions}

In conclusion, we have carried out systematic measurements of the nonlinear optical transmission of three series of ferrite core/shell nanoparticles with different shell thicknesses $\left(\mathrm{MnFe}_{2} \mathrm{O}_{4} / \mathrm{CoFe}_{2} \mathrm{O}_{4}\right.$, and $\mathrm{CoFe}_{2} \mathrm{O}_{4} / \mathrm{MnFe}_{2} \mathrm{O}_{4}$-with two different core sizes-), excited by ultrashort (100 fs) laser pulses at $800 \mathrm{~nm}$. The results show that the effective two-photon absorption coefficient and the effective third order susceptibility reach maximum values for relatively small shell thicknesses, for all the three series. FDTD simulations reveal that the core/shell morphology improves local electric field enhancement, particularly for the smaller cores. The optical limiting performance (limiting threshold fluence in the range of $1.66 \mathrm{~J} / \mathrm{cm}^{2}$ ) and photostability (apparent lack of photodamage up to $35 \mathrm{TW} / \mathrm{cm}^{2}$ in liquid dispersions) of these ferrite core/shell nanoparticles is high, compared to most of the recently investigated materials and benchmark materials like $\mathrm{C}_{60}$ or carbon nanotubes. In fact, the robustness of the physiochemical properties of ferrite materials, even in harsh environments, together with the outstanding optical limiting efficiency of ferrite core/shell nanoparticles, make them very 
promising materials for fabricating optical limiters, for protecting human eyes and optical sensors from hazardous laser radiation.

\section{Experimental Section}

The synthesis was carried out using standard airless procedures and commercially available reagents: 1-octadecene (ODE, 90\%), ethanol $(\mathrm{EtOH},>99.8 \%)$, hexane $(\mathrm{Hx},>95 \%)$, oleic acid (OAc, 90\%), Oleylamine (OAm, $70 \%$ ), iron(III) acetylacetonate $\left(\mathrm{Fe}(\mathrm{acac})_{3},>97 \%\right)$, cobalt(II) acetylacetonate $\left(\mathrm{Co}(\mathrm{acac})_{2},>97 \%\right)$. All starting materials were purchased from Sigma-Aldrich.

Monodisperse spherical NPs were synthesized through thermal decomposition of the metalsurfactant complex in a high-boiling solvent containing OAc and OAm as stabilizing surfactant. ${ }^{[67]}$ Briefly, in order to synthesize $6 \mathrm{~nm} \mathrm{MnFe}{ }_{2} \mathrm{O}_{4}$ nanoparticles $\mathrm{Mn}(\mathrm{acac})_{2}(3.25$ $\mathrm{mmol})$ and $\mathrm{Fe}(\mathrm{acac})_{3}(5 \mathrm{mmol})$ were placed in a $50 \mathrm{~mL}$ three-neck round-bottom flask in the presence of Oac $(6 \mathrm{mmol})$, Oam $(6 \mathrm{mmol})$ and $20 \mathrm{~mL}$ of $\mathrm{Bz}_{2} \mathrm{O}$. The reaction mixture was heated at $200^{\circ} \mathrm{C}$ for 2 hour and posteriorly at $300{ }^{\circ} \mathrm{C}$ for $1 \mathrm{~h}$ and, after removing the heat source, the reaction products were cooled to room temperature. Posteriorly $40 \mathrm{mg}$ of assynthesized $\mathrm{MnFe}_{2} \mathrm{O}_{4}$ nanoparticles were mixed with $\mathrm{Co}(\mathrm{acac})_{2}(3.25 \mathrm{mmol}), \mathrm{Fe}(\mathrm{acac})_{3}(5$ mmol) Oac $(6 \mathrm{mmol})$, Oam $(6 \mathrm{mmol})$ and $20 \mathrm{~mL}$ of $\mathrm{Bz}_{2} \mathrm{O}$ and heated to $300{ }^{\circ} \mathrm{C}$ during 1 hour for the growth of a $1.5 \mathrm{~nm}$ Co-ferrite shell. Larger Co-ferrite shell thickness was obtained repeating the same procedure using Mn-ferrite/Co-ferrite core/shell nanoparticles as a seeds. All NPs were washed by several cycles of coagulation with ethanol, centrifugation at 5000 rpm, disposal of supernatant solution and re-dispersion in hexane.

TEM images were obtained using a Jeol JEM-2010 microscope with a LaB6 filament operated at $200 \mathrm{kV}$. HAADF images and EELS analysis were performed in a FEI Tecnai F20 equipped with a Quantum GIF EELS spectrometer. 
XRD patterns were collected using a Panalytical X'Pert Pro diffractometer with $\mathrm{Cu} \mathrm{K} \alpha$ radiation. The measurements were carried out in the range $25-70^{\circ}$, with a step size of $0.03^{\circ}$ and a collection time of $1.5 \mathrm{~s}$. Quantitative analysis of the XRD data was performed with a full pattern fitting procedure based on the fundamental parameter approach (Rietveld method) using MAUD software. ${ }^{[68]}$

The magnetic properties of the nanoparticles were measured on tightly packed powdered samples using a superconducting quantum interference device (SQUID, Quantum Design) magnetometer with $70 \mathrm{kOe}$ maximum field. The magnetization versus temperature measurements were performed in zero field cooled (ZFC) conditions in 50 Oe.

The open-aperture Z-scan technique was used for measuring the nonlinear optical transmittance of the samples. In this method, the nonlinear transmission of light through the material is measured as a function of input fluence (energy per unit area) or intensity (fluence divided by laser pulse width). The input light fluence (and hence the intensity) is varied by moving the sample through the focal region of a focused laser beam. A regeneratively amplified Ti:Sapphire laser (TSA-10, Spectra Physics) producing 100 fs laser pulses at 800 $\mathrm{nm}$, at a repetition rate of $10 \mathrm{~Hz}$, was used for excitation. An electronically synchronized fast mechanical shutter was used in the beam path to select single pulses from the $10 \mathrm{~Hz}$ pulse train so that at the sample, there was approximately 5 seconds interval between each successive laser shot. By using a beam expander and a diamond aperture the spatial profile of the laser beam was further improved to provide a near-perfect Gaussian (TEM 00 ) mode. The laser pulse energy used was $10 \mu \mathrm{J}$. A plano-convex lens having a focal length of $10.75 \mathrm{~cm}$ was used to focus the laser beam, which gives a focal spot radius of about 24 microns. Samples uniformly dispersed in toluene by ultra-sonication were taken in $1 \mathrm{~mm}$ path length glass cuvettes for the measurement. Concentrations were adjusted such that all samples had a linear transmission of $70 \%$ at the excitation wavelength. By mounting the sample cuvette on a 
precision translation stage, fine movement along the $\mathrm{z}$-direction through the beam focus was facilitated (focal point is taken as $\mathrm{z}=0$ ). The experiment was automated using a LabVIEW program, which controlled the movement of the translation stage, activation of the shutter, data acquisition from the oscilloscope (to which the energy meters are connected) and storage in a computer. A plot of the sample transmission against sample position gives the open aperture Z-scan curve. Following convention, the transmission is normalized to the linear transmission measured at low input intensities (corresponding to large $\mathrm{z}$ values). For example, if the linear transmission measured at low input intensity is $70 \%$, and the transmission drops to $35 \%$ at the beam focus (due to the nonlinearity), the normalized transmission at the focus is $35 / 70=0.5$. From the Z-scan curve it is possible to plot the sample transmission against input laser fluence or intensity since for a spatially Gaussian beam, the light fluence $F_{\text {in }}(z)$ at any position $z$ can be calculated from the corresponding beam radius $\omega(\mathrm{z})$ and the input laser pulse energy $E_{i n}$. The beam radius is given by ${ }^{[69]}$

$$
\omega(z)=\omega(0)\left[1+\left(z / z_{0}\right)^{2}\right]^{1 / 2},
$$

the position-dependent fluence is given by ${ }^{[69]}$

$$
\mathrm{F}_{\text {in }}(\mathrm{z})=4(\ln 2)^{1 / 2} \mathrm{E}_{\mathrm{in}} / \pi^{3 / 2} \omega(\mathrm{z})^{2},
$$

and the position dependent intensity is given by ${ }^{[69]}$

$$
\mathrm{I}_{\text {in }}(\mathrm{z})=\mathrm{F}_{\text {in }}(\mathrm{z}) / \tau \text {, }
$$

where $\omega(0)$ is the focal spot radius, $\mathrm{z}_{0}=\pi \omega(0)^{2} / \lambda$ is the Rayleigh range, and $\tau$ is the laser pulsewidth.

\section{Supporting Information}

Supporting Information is available from the Wiley Online Library or from the author.

\section{Acknowledgements}

We acknowledge the support of the 2014-SGR-1015 project of the Generalitat de Catalunya. ALO acknowledges the Spanish Ministrio de Economía y Competitividad through the Juan de la Cierva Program (IJCI-2014-21530). ). ICN2 is founded by the CERCA 
Programme/Generalitat de Catalunya. ICN2 also acknowledges support from the Severo Ochoa Program (MINECO, Grant SEV-2013-0295). RP thanks Ms. Nivya Roy for technical help with some of the measurements. SP thanks Ms. Sarayoo Sasidharan Kangaparambil for useful discussions. ALO thanks Dr. Christopher Tollan for the critical reading of the manuscript.

\section{References}

[1] S. Perumbilavil, P. Sankar, T. Priya Rose, R. Philip, Appl. Phys. Lett. 2015, 107, 51104.

[2] P. Chen, X. Wu, X. Sun, J. Lin, W. Ji, K. L. Tan, Phys. Rev. Lett. 1999, 82, 2548.

[3] B. Anand, A. Kaniyoor, D. Swain, T. T. Baby, S. Venugopal Rao, S. S. Sankara Sai, S. Ramaprabhu, R. Philip, J. Mater. Chem. C 2014, 2, 10116.

[4] R. P. Ummer, P. Sreekanth, B. Raneesh, R. Philip, D. Rouxel, S. Thomas, N. Kalarikkal, RSC Adv. 2015, 5, 67157.

[5] P. Thomas, P. Sreekanth, K. E. Abraham, J. Appl. Phys. 2015, 117, 53103.

[6] K. A. Ann Mary, N. V. Unnikrishnan, R. Philip, RSC Adv. 2015, 5, 14034.

[7] K. A. A. Mary, N. V. Unnikrishnan, R. Philip, APL Mater. 2014, 2, 76104.

[8] G. S. He, K.-T. Yong, Q. Zheng, Y. Sahoo, A. Baev, A. I. Ryasnyanskiy, P. N. Prasad, Opt. Express 2007, 15, 12818.

[9] B. S. Kalanoor, P. B. Bisht, S. Akbar Ali, T. T. Baby, S. Ramaprabhu, J. Opt. Soc. Am. B 2012, 29, 669.

[10] B. Anand, A. Kaniyoor, S. S. S. Sai, R. Philip, S. Ramaprabhu, J. Mater. Chem. C 2013, 1, 2773.

[11] A. S. Aricò, P. Bruce, B. Scrosati, J.-M. Tarascon, W. van Schalkwijk, Nat. Mater. 2005, $4,366$.

[12] A. López-Ortega, M. Estrader, G. Salazar-Alvarez, A. G. Roca, J. Nogués, Phys. Rep. 2015, 553, 1 .

[13] A. López-Ortega, E. Lottini, C. de Julián Fernández, C. Sangregorio, Chem. Mater. 2015, 27, 4048.

[14] K. L. Kelly, E. Coronado, L. L. Zhao, G. C. Schatz, J. Phys. Chem. B 2003, 107, 668.

[15] M. Quinten, Optical Properties of Nanoparticle Systems: Mie and Beyond., Wiley Online Library, 2011.

[16] L. M. Liz-Marzán, P. Mulvaney, J. Phys. Chem. B 2003, 107, 7312.

[17] H. Althues, J. Henle, S. Kaskel, Chem. Soc. Rev. 2007, 36, 1454.

[18] X. Zhang, B. Sun, R. H. Friend, H. Guo, D. Nau, H. Giessen, Nano Lett. 2006, 6, 651.

[19] M. Ando, K. Kadono, M. Haruta, T. Sakaguchi, M. Miya, Nature 1995, 374, 625.

[20] K. Sridharan, P. Sreekanth, T. J. Park, R. Philip, J. Phys. Chem. C 2015, 119, 16314.

[21] K. Sridharan, M. S. Ollakkan, R. Philip, T. J. Park, Carbon N. Y. 2013, 63, 263.

[22] T. J. Wagner, M. J. Bohn, R. A. Coutu, Jr., L. P. Gonzalez, J. M. Murray, K. L. Schepler, S. Guha, J. Opt. Soc. Am. B 2010, 27, 2122.

[23] A. A. Manenkov, Opt. Eng. 2014, 53, 10901.

[24] A. Goldman, Modern Ferrite Technology, 2nd Ed., Springer Berlin Heidelberg, 2006.

[25] M. O. Ramirez, A. Kumar, S. A. Denev, N. J. Podraza, X. S. Xu, R. C. Rai, Y. H. Chu, J. Seidel, L. W. Martin, S.-Y. Yang, E. Saiz, J. F. Ihlefeld, S. Lee, J. Klug, S. W. Cheong, M. J. Bedzyk, O. Auciello, D. G. Schlom, R. Ramesh, J. Orenstein, J. L. Musfeldt, V. Gopalan, Phys. Rev. B 2009, 79, 224106.

[26] S. S. Nair, J. Thomas, C. S. Suchand Sandeep, M. R. Anantharaman, R. Philip, Appl. Phys. Lett. 2008, 92, 171908. 
[27] J. J. Thomas, S. Krishnan, K. Sridharan, R. Philip, N. Kalarikkal, Mater. Res. Bull. 2012, 47, 1855.

[28] P. Chantharasupawong, R. Philip, T. Endo, J. Thomas, Appl. Phys. Lett. 2012, 100, 221108.

[29] J. Nogués, J. Sort, V. Langlais, V. Skumryev, S. Suriñach, J. S. Muñoz, M. D. Baró, Phys. Rep. 2005, 422, 65.

[30] R. Ghosh Chaudhuri, S. Paria, Chem. Rev. 2012, 112, 2373.

[31] K. S. Kumar, V. B. Kumar, P. Paik, J. Nanoparticles 2013, 2013, 1.

[32] A. Juhin, A. López-Ortega, M. Sikora, C. Carvallo, M. Estrader, S. Estradé, F. Peiró, M. D. Baró, P. Sainctavit, P. Glatzel, J. Nogués, Nanoscale 2014, 6, 11911.

[33] A. Quesada, C. Granados-Miralles, A. López-Ortega, S. Erokhin, E. Lottini, J. Pedrosa, A. Bollero, A. M. Aragón, F. Rubio-Marcos, M. Stingaciu, G. Bertoni, C. de Julián Fernández, C. Sangregorio, J. F. Fernández, D. Berkov, M. Christensen, Adv. Electron. Mater. 2016, 2, 1500365.

[34] M. Cabo, E. Pellicer, E. Rossinyol, M. Estrader, A. López-Ortega, J. Nogués, O. Castell, S. Suriñach, M. D. Baró, J. Mater. Chem. 2010, 20, 7021.

[35] J. J. Schneider, Adv. Mater. 2001, 13, 529.

[36] Y. Lu, Y. Yin, Z.-Y. Li, Y. Xia, Nano Lett. 2002, 2, 785.

[37] J. A. De Toro, D. P. Marques, P. Muñiz, V. Skumryev, J. Sort, D. Givord, J. Nogués, Phys. Rev. Lett. 2015, 115, 57201.

[38] G. Carotenuto, G. P. Pepe, L. Nicolais, Eur. Phys. J. B 2000, 16, 11.

[39] F. Wang, R. Deng, J. Wang, Q. Wang, Y. Han, H. Zhu, X. Chen, X. Liu, Nat. Mater. 2011, $10,968$.

[40] J.-H. H. Lee, J.-T. T. Jang, J.-S. S. Choi, S. H. Moon, S.-H. H. Noh, J.-G. G. J.-W. W. Kim, J.-G. G. J.-W. W. Kim, I.-S. S. Kim, K. I. Park, J. W. Cheon, Nat. Nanotechnol. 2011, 6, 418.

[41] M. Estrader, A. López-Ortega, S. Estradé, I. V Golosovsky, G. Salazar-Alvarez, M. Vasilakaki, K. N. Trohidou, M. Varela, D. C. Stanley, M. Sinko, M. J. Pechan, D. J. Keavney, F. Peiró, S. Suriñach, M. D. Baró, J. Nogués, Nat. Commun. 2013, 4, 2960.

[42] M. Estrader, A. López-Ortega, I. V Golosovsky, S. Estradé, A. G. Roca, G. SalazarAlvarez, L. López-Conesa, D. Tobia, E. Winkler, J. D. Ardisson, W. A. A. Macedo, A. Morphis, M. Vasilakaki, K. N. Trohidou, A. Gukasov, I. Mirebeau, O. L. Makarova, R. D. Zysler, F. Peiró, M. D. Baró, L. Bergström, J. Nogués, Nanoscale 2015, 7, 3002.

[43] E. Lottini, A. López-Ortega, G. Bertoni, S. Turner, M. Meledina, G. Van Tendeloo, C. de Julián Fernández, C. Sangregorio, Chem. Mater. 2016, 28, 4214.

[44] M. Acebrón, J. F. Galisteo-López, D. Granados, J. López-Ogalla, J. M. Gallego, R. Otero, C. López, B. H. Juárez, ACS Appl. Mater. Interfaces 2015, 7, 6935.

[45] A. E. Neeves, M. H. Birnboim, J. Opt. Soc. Am. B 1989, 6, 787.

[46] Y. Yang, M. Nogami, J. Shi, H. Chen, Y. Liu, S. Qian, J. Mater. Chem. 2003, 13, 3026.

[47] R. Weissleder, Nat. Biotechnol. 2001, 19, 316.

[48] S. Estradé, L. Yedra, A. López-Ortega, M. Estrader, G. Salazar-Alvarez, M. D. D. Baró, J. Nogués, F. Peiró, Micron 2012, 43, 30.

[49] L. Yedra, E. Xuriguera, M. Estrader, A. López-Ortega, M. D. Baró, J. Nogués, M. Roldan, M. Varela, S. Estradé, F. Peiró, Microsc. Microanal. 2014, 20, 698.

[50] A. Lopez-Santiago, H. R. Grant, P. Gangopadhyay, R. Voorakaranam, R. A. Norwood, N. Peyghambarian, Opt. Mater. Express 2012, 2, 978.

[51] B. Anand, R. Podila, P. Ayala, L. Oliveira, R. Philip, S. S. S. Sai, A. A. Zakhidov, A. M. Rao, Nanoscale 2013, 5, 7271.

[52] M. Rumi, J. W. Perry, Adv. Opt. Photonics 2010, 2, 451.

[53] E. W. Van Stryland, M. Sheik-Bahae, in Z-Scan Meas. Opt. Non-Linearities (Eds: 
M.G. Kuzyk, C.W. Dirik), Marcel Dekker, Inc., New York, 1998, 1998, pp. 655-692.

[54] R. L. Sutherland, Handbook of Nonlinear Optics, New York, 2003.

[55] B.-H. Zhu, H.-C. Zhang, Z.-Y. Zhang, Y.-P. Cui, J.-Y. Zhang, Appl. Phys. Lett. 2011, 99, 231903.

[56] C. Gan, M. Xiao, D. Battaglia, N. Pradhan, X. Peng, Appl. Phys. Lett. 2007, 91, 201103.

[57] Y. Yang, M. Hori, T. Hayakawa, M. Nogami, Surf. Sci. 2005, 579, 215.

[58] J. P. Huang, K. W. Yu, Phys. Rep. 2006, 431, 87.

[59] J. Zhang, X. Zhang, J. Y. Zhang, J. Phys. Chem. C 2010, 114, 3904.

[60] B. H. Zhu, H. C. Zhang, J. Y. Zhang, Y. P. Cui, Z. Q. Zhou, Appl. Phys. Lett. 2011, 99, 21908.

[61] S. A. Salman, Z. T. Khodair, S. J. Abed, Int. Lett. Chem. Phys. Astron. 2015, 61, 118.

[62] S. S. Batsanov, E. D. Ruchkin, I. A. Poroshina, Refractive Indices of Solids, Springer Singapore, Singapore, 2016.

[63] L. Lamaignre, in Adv. Top. Meas. (Ed: M.Z. Haq), InTech, 2012, pp. 61-78.

[64] A. Stratan, A. Zorila, L. Rusen, S. Simion, C. Blanaru, C. Fenic, L. Neagu, G. Nemes, in Laser-Induced Damage Opt. Mater. 2012 (Eds: G.J. Exarhos, V.E. Gruzdev, J.A. Menapace, D. Ristau, M.J. Soileau), SPIE Proceedings, 2012, p. 85301Y.

[65] A. M. Summers, A. S. Ramm, G. Paneru, M. F. Kling, B. N. Flanders, C. A. TralleroHerrero, Opt. Express 2014, 22, 4235.

[66] K. A. Janulewicz, A. Hapiddin, D. Joseph, K. E. Geckeler, J. H. Sung, P. V. Nickles, Appl. Phys. A 2014, 117, 1811.

[67] S. Sun, H. Zeng, J. Am. Chem. Soc. 2002, 124, 8204.

[68] L. Lutterotti, "MAUD program," can be found under http://www.ing.unitn.it/ maud/, 2016.

[69] B. E. A. Saleh, M. C. Teich, Fundamentals of Photonics, Wiley-Interscience, 2007. 

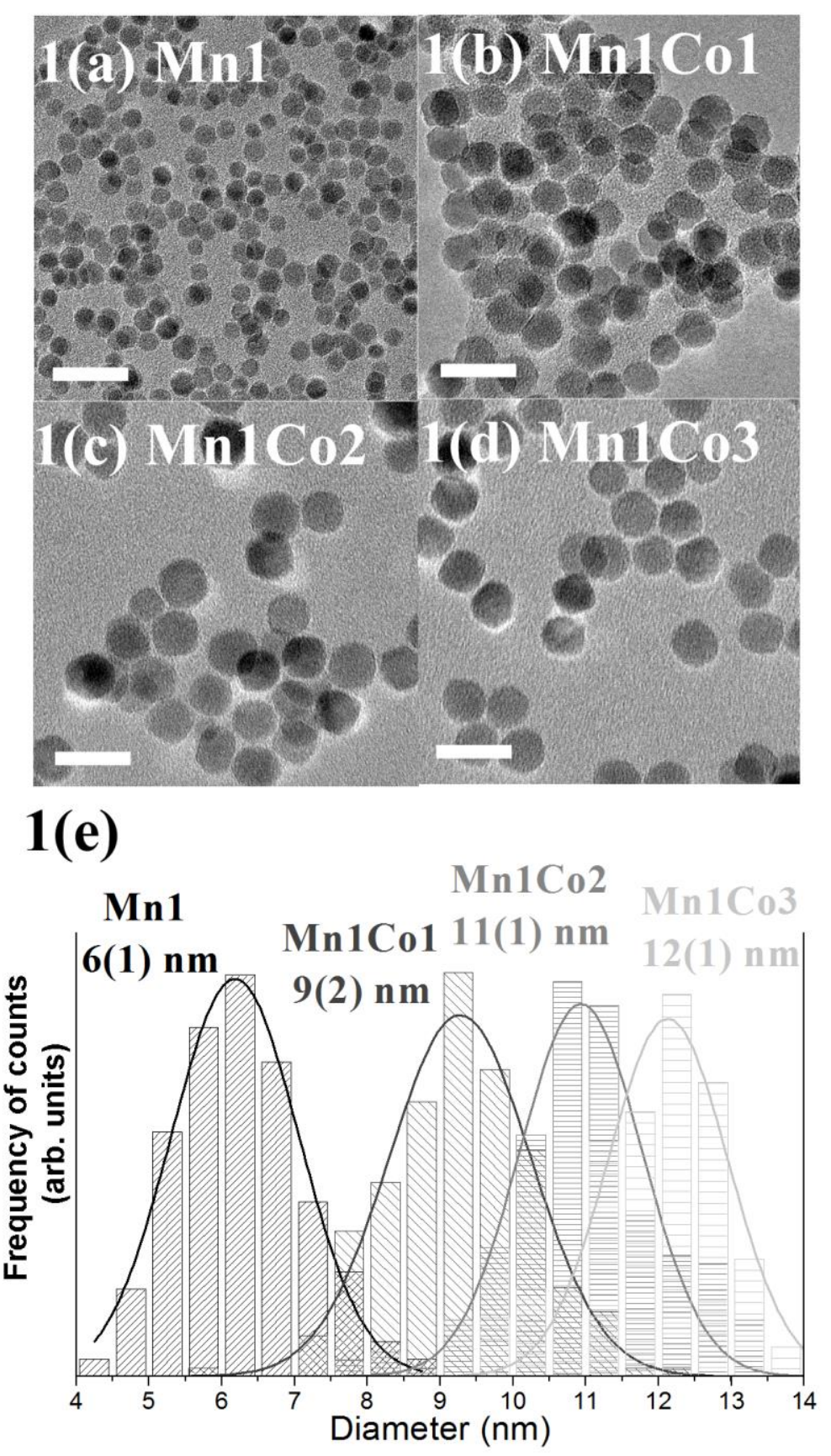

Figure 1. (a-d) TEM images and (e) their respective particle size histograms for Mn1@Co core/shell nanoparticle series. Scale bars correspond to a length of $20 \mathrm{~nm}$. 


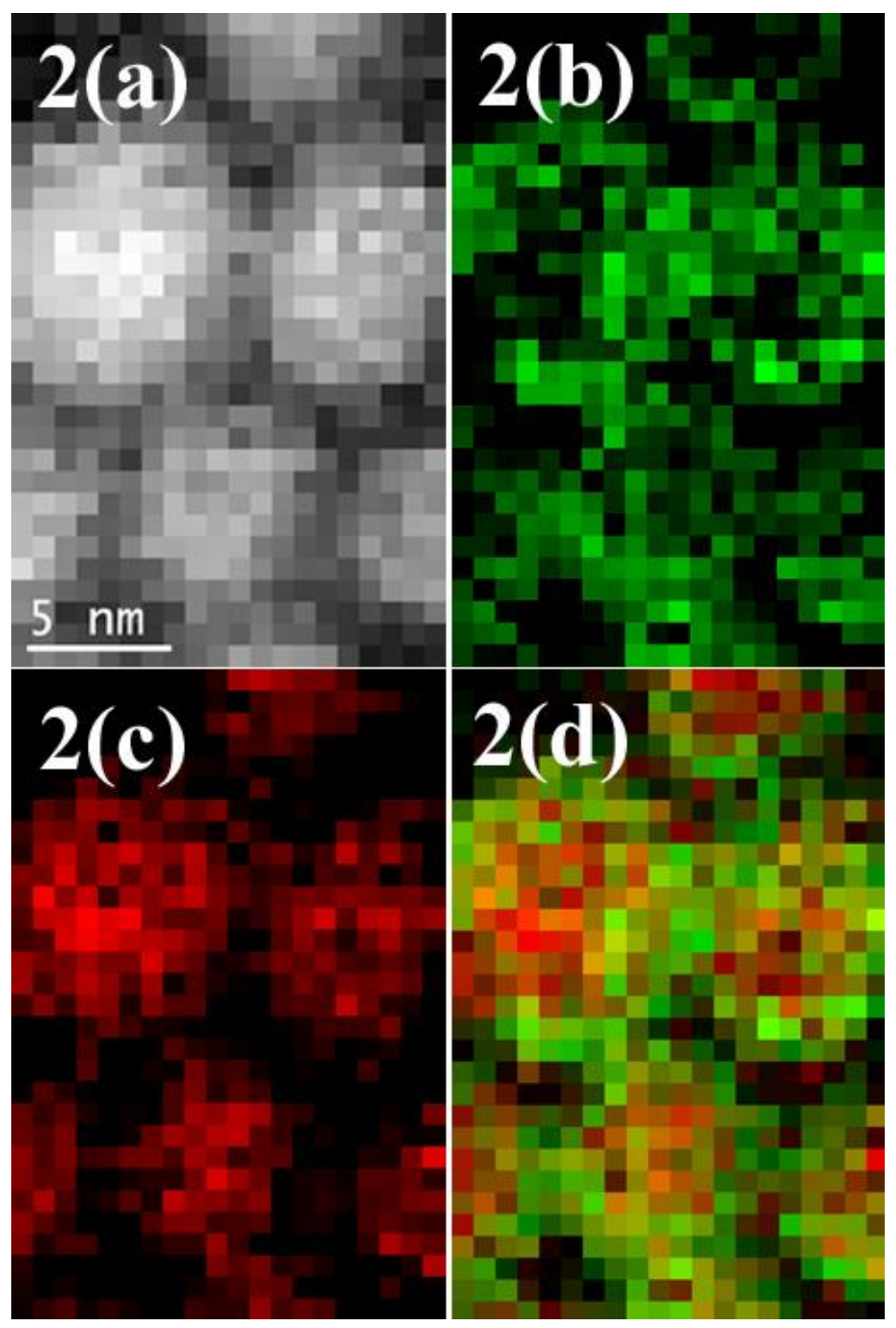

Figure 2. (a) High-angle annular dark-field (HAADF) TEM image for sample Co1@Mn3 and their respective (b) $\mathrm{Mn}$ and (c) Co maps of integrated $\mathrm{L}_{2,3}$ edge signal. (d) Co/Mn EELS mapping reconstruction (red and green colors refer to cobalt and manganese L edge signal, respectively). 


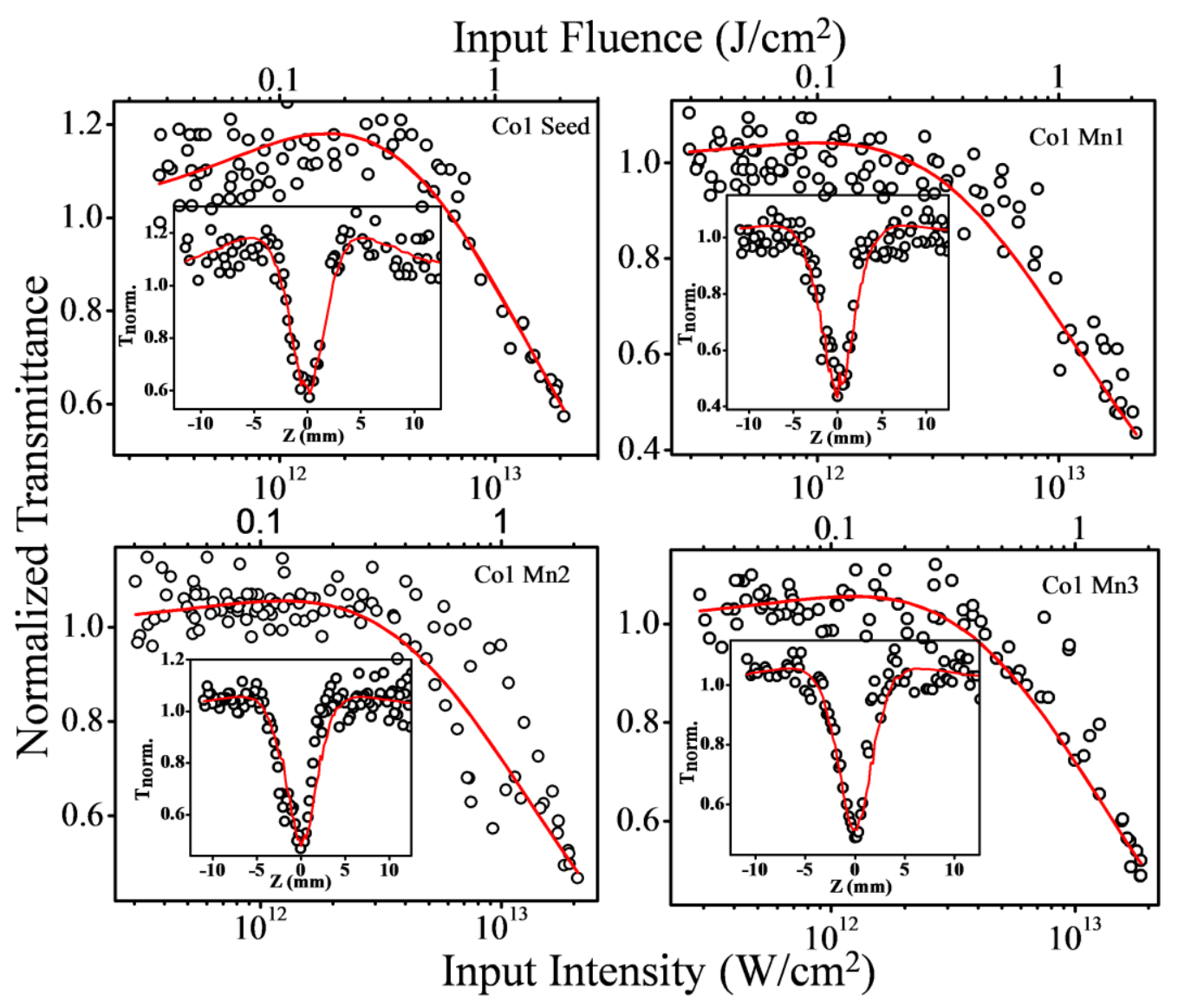

Figure 3. Normalized optical transmittance (for $800 \mathrm{~nm}, 10 \mu \mathrm{J}, 100 \mathrm{fs}$ pulses) obtained for the Co1@Mn series as a function of input intensity, calculated from the Z-scan curves (shown in the insets). The input fluence values also are shown. Symbols are experimental data, while the solid curves are numerical fits obtained using Equation (2), from which the $\beta$ and $I_{s}$ values are determined. 


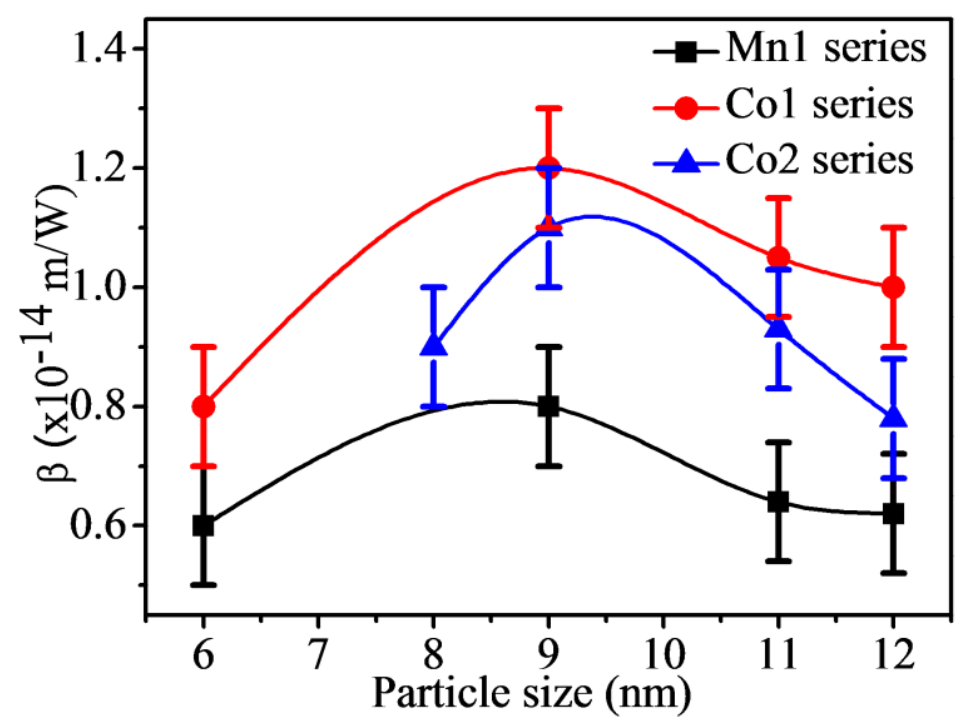

Figure 4. Variation of $\beta$ as a function of particle size. The vertical lines are the error bars. The solid curves are guides to the eye.
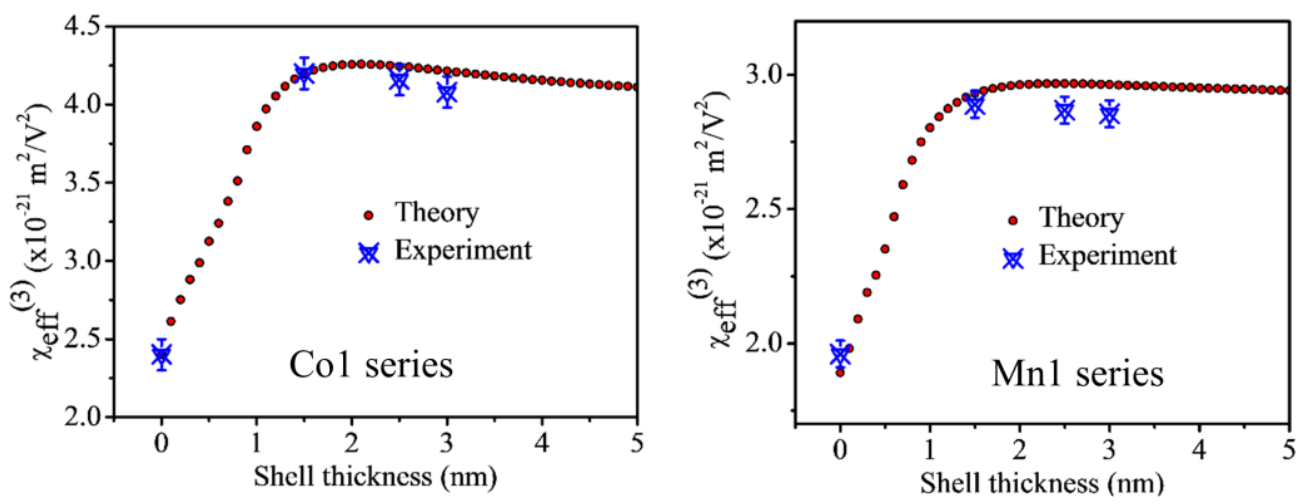

Figure 5. Theoretically calculated (using Equation 4) and experimentally estimated effective nonlinear susceptibility $\left(\chi_{\mathrm{eff}^{(3)}}\right)$ values for the Co1 and Mn1 series for different shell thicknesses. 

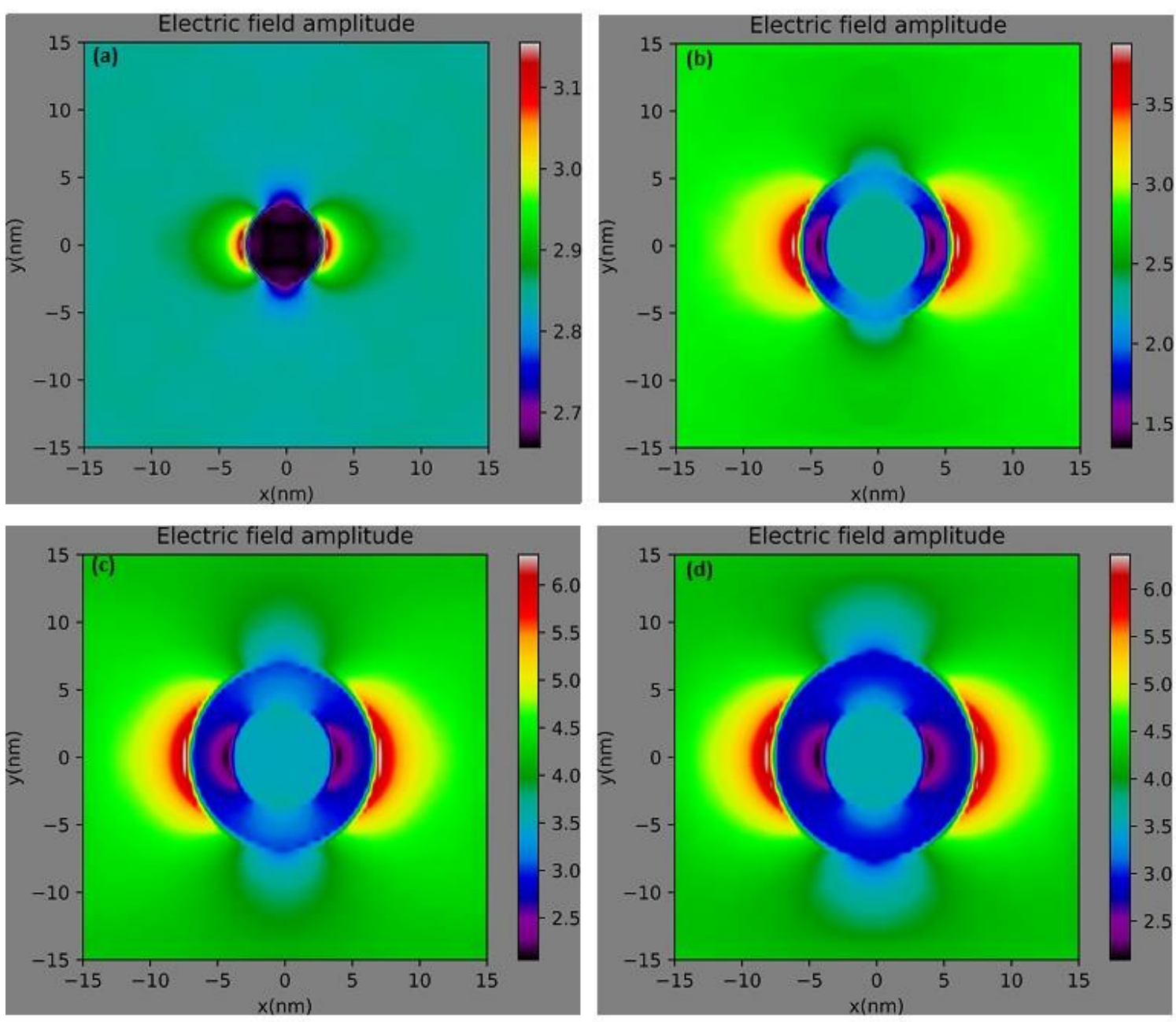

Figure 6. FDTD simulation results for (a) Co1 and (b,c,d) Co1@Mn nanoparticles periodically dispersed in the dielectric medium toluene, with an inter-particle separation of 30 $\mathrm{nm}$. The electric field amplitude within the nanoparticle and in its vicinity is plotted in a color scale, where the input field has been taken as unity. The core diameter is $6 \mathrm{~nm}$ for all samples, while the shell thickness is $0 \mathrm{~nm}, 1.5 \mathrm{~nm}, 2.5 \mathrm{~nm}$ and $3 \mathrm{~nm}$ for a, b, c, and d, respectively. 
Ultrashort laser pulse (100 fs) excitation of spinel based core/shell nanoparticles reveals a non-monotonic variation of the effective two-photon absorption coefficient, with a maximum value seen for relatively thin shells. Considering local field enhancement effects, nonlinear transmission studies and FDTD simulations indicate that core/shell is an advantageous morphology to improve the nonlinearity of the composite for applications like ultrafast optical limiting.

Keywords: nonlinear absorption; core/shell nanoparticles; optical limiting; oxide heterostructures; FDTD simulations

Sreekanth Perumbilavil, Alberto López-Ortega.* Gaurav Kumar Tiwari , Josep Nogués, Tamio Endo, Reji Philip

Enhanced Ultrafast Nonlinear Optical Response in Ferrite Core/Shell Nanostructures with Excellent Optical Limiting Performance

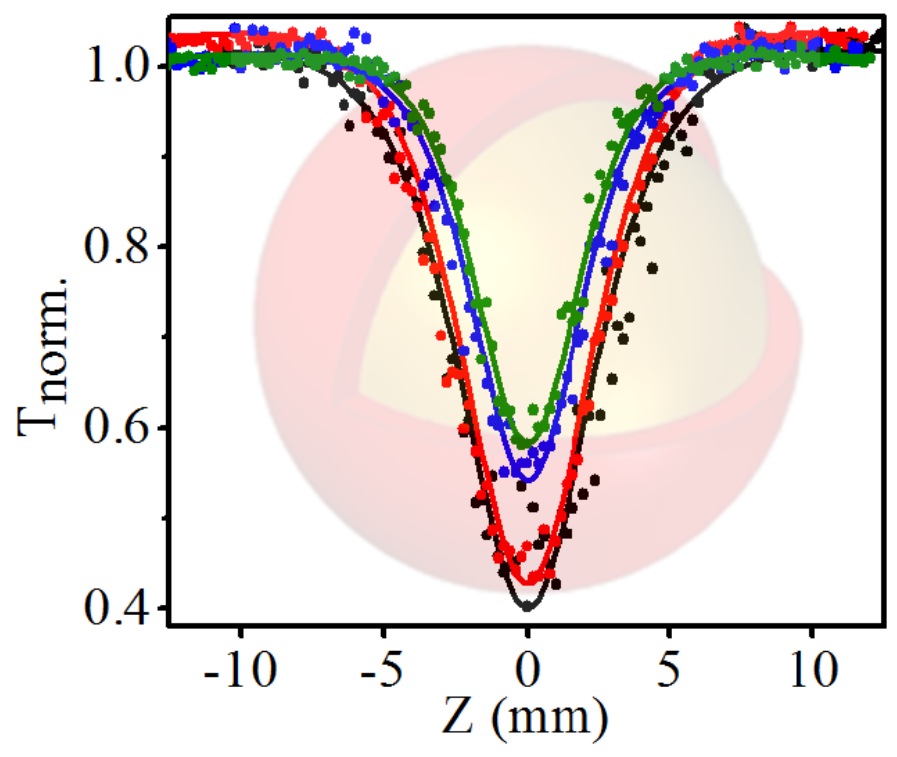


Copyright WILEY-VCH Verlag GmbH \& Co. KGaA, 69469 Weinheim, Germany, 2016.

\section{Supporting Information}

\section{Enhanced Ultrafast Nonlinear Optical Response in Ferrite Core/Shell Nanostructures with Excellent Optical Limiting Performance}

Sreekanth Perumbilavil, Alberto López-Ortega*, Josep Nogués, Tamio Endo, Reji Philip
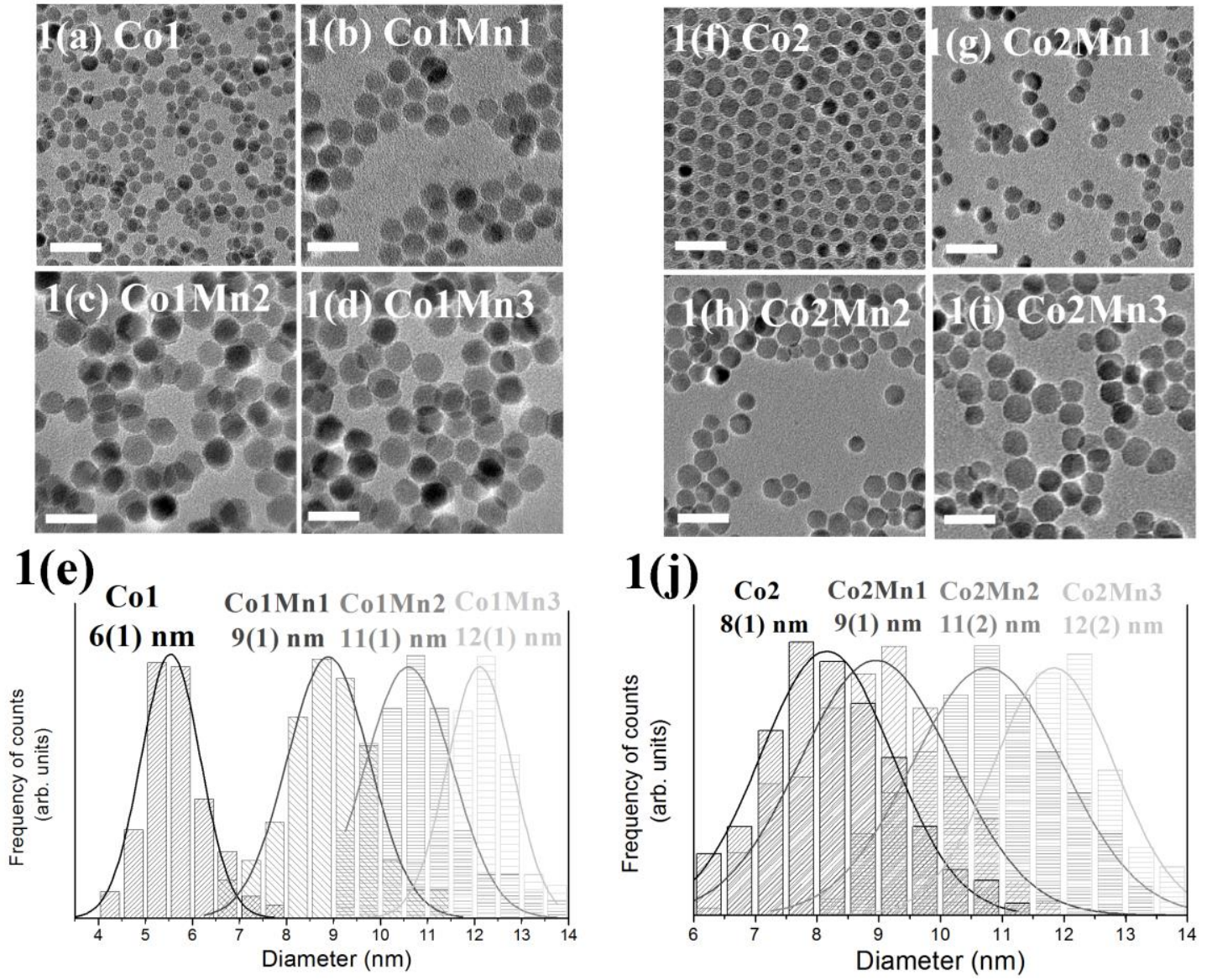

Figure S1.(a-d, f-i) TEM images and (e, j) their respective particle size histograms for Co1@Mn and Co2@Mncore/shell nanoparticle series. Scale bars correspond to a length of $20 \mathrm{~nm}$. 


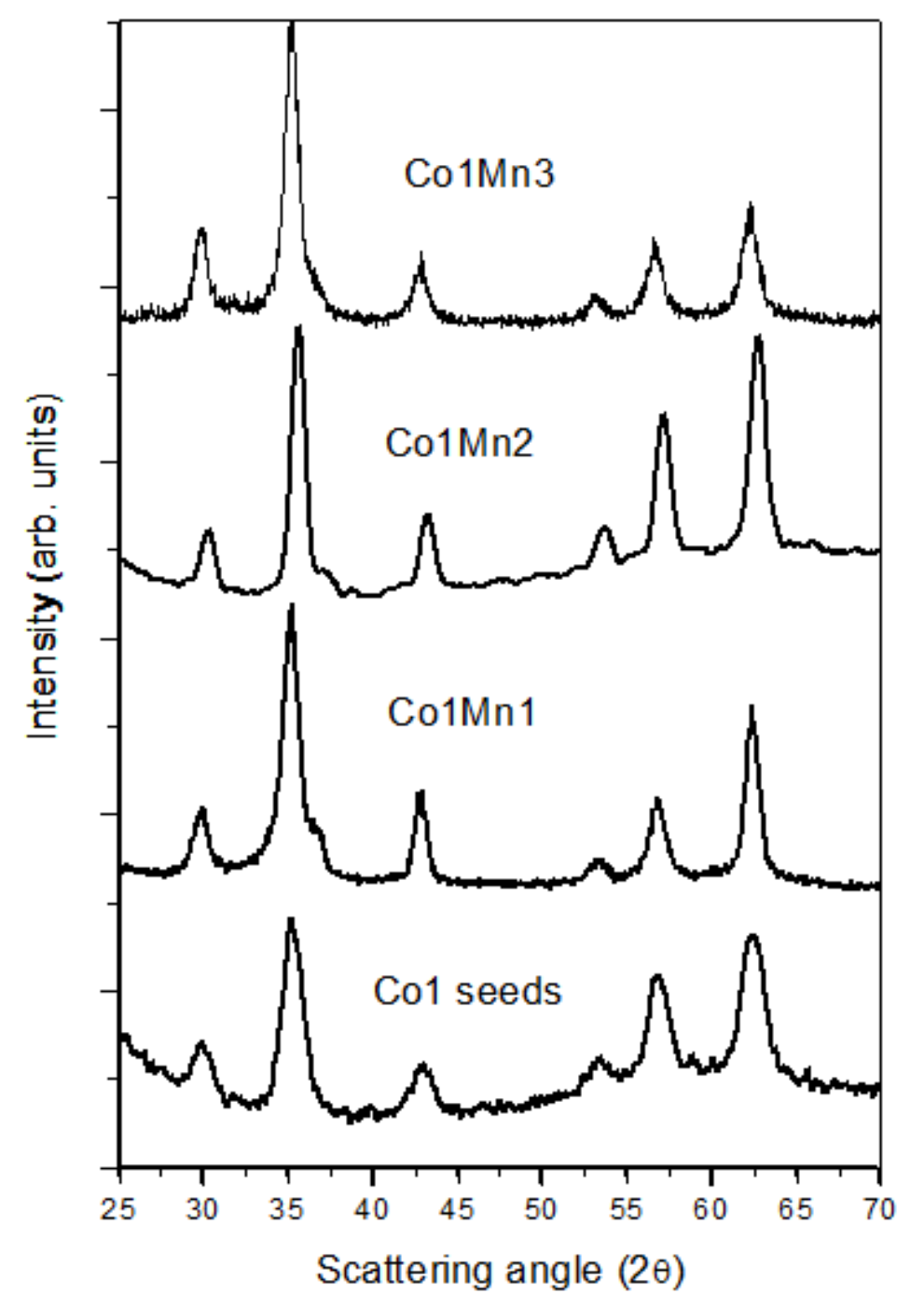

Figure S2. XRD diffraction patterns for the series of Co1 nanoparticles. 


\section{Magnetic Characterization}

As can be seen in Figure S3a, as the shell thickness increases the blocking temperature, $\mathrm{T}_{\mathrm{B}}$, of the nanoparticles (estimated form the maximum of the temperature dependence of the magnetization, $\mathrm{M}(\mathrm{T})$ ) becomes higher. Since the blocking temperature is proportional to the effective anisotropy, $\mathrm{K}_{\mathrm{Eff}}$, and the volume, $\mathrm{V}\left(\mathrm{T}_{\mathrm{B}} \propto \mathrm{K}_{\mathrm{Eff}} \mathrm{V}\right)$, we can readily obtain $\mathrm{K}_{\mathrm{Eff}}$ from the data in the inset of Figure S3b. It can be observed that $\mathrm{K}_{\mathrm{Eff}}$ decreases rather fast as the shell thickness increases (see Figure S3b). This is expected since the core is composed of Co-ferrite with a high anisotropy $\left(\mathrm{K}_{\text {Co-ferrite }} \sim 10^{6} \mathrm{erg} / \mathrm{cm}^{3}\right)$, while the shell is a soft ferrimagnet $\left(\mathrm{K}_{\mathrm{Mn} \text {-ferrite }}\right.$ $\sim 10^{4} \mathrm{erg} / \mathrm{cm}^{3}$ ). In fact, if the thickness of the soft counterpart is sufficiently thin (as in the case of core/shell structures), $\mathrm{K}_{\mathrm{Eff}}$ when coupling a hard and a soft material is given by $\mathrm{K}_{\mathrm{Eff}}=$ $f_{\text {soft }} \mathrm{K}_{\text {soft }}+f_{\text {hard }} K_{\text {hard }}$, where $f_{\text {soft }}$ and $f_{\text {hard }}$ are the volume fractions of the soft and hard materials, respectively. ${ }^{[1]}$ Given that $\mathrm{K}_{\mathrm{Mn} \text {-ferrite }}<<\mathrm{K}_{\text {Co-ferrite, }} \mathrm{K}_{\mathrm{Eff}}$ becomes $\mathrm{K}_{\mathrm{Eff}}=f_{\text {hard }} \mathrm{K}_{\text {hard }}$, where $f_{\text {hard }}$ is given by the core/shell geometry: $f_{\text {hard }}=\mathrm{V}_{\text {core }} / \mathrm{V}_{\text {tot }}=\mathrm{R}_{\text {core }}{ }^{3} /\left(\mathrm{t}_{\text {Shell }}+\mathrm{R}_{\text {Core }}\right)^{3}$. Consequently, since $\mathrm{R}_{\text {core }}$ is constant for all samples, if the samples have a core/shell morphology we would expect $\mathrm{K}_{\text {Eff }}$ to decay as $1 /\left(\mathrm{t}_{\text {Shell }}+\mathrm{R}_{\mathrm{Core}}\right)^{3}$ as $\mathrm{t}_{\text {Shell }}$ increases. Indeed, as shown by the continuous line in Fig. S3(b) the decrease of $K_{\text {Eff }}$ follow nicely the expected $1 /\left(t_{\text {Shell }}+\mathrm{R}_{\text {Core }}\right)^{3}$ behaviour, thus confirming the core/shell architecture. 

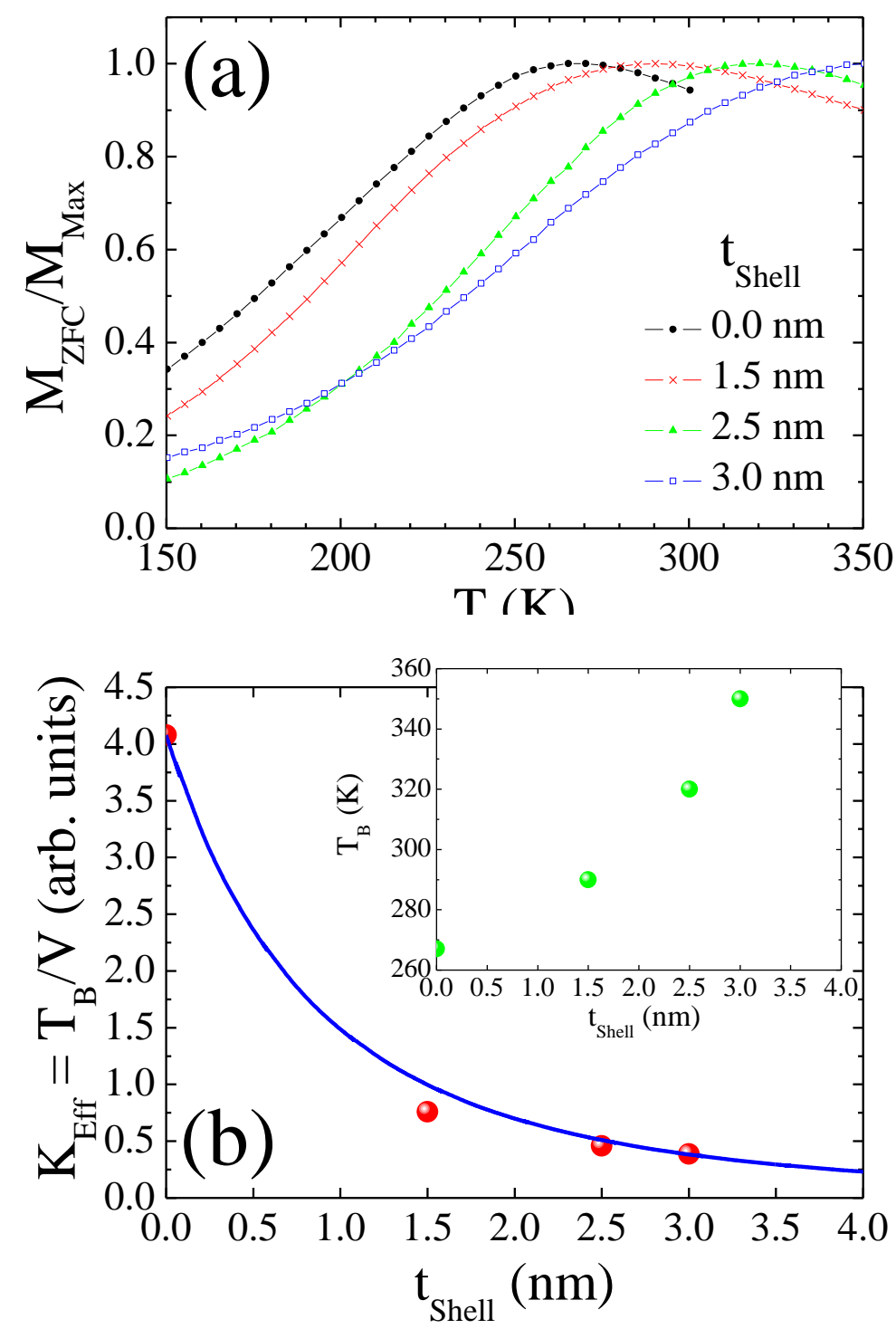

Figure S3. (a) Temperature dependence of the normalized zero field cooled magnetization $\left(\mathrm{M}_{\mathrm{ZFC}} / \mathrm{M}_{\mathrm{Max}}\right)$ for a series of core/shell nanoparticles with a Co-ferrite core with a radius $\mathrm{R}_{\mathrm{Core}}$ $=2.5 \mathrm{~nm}$ and different Mn-ferrite shells with thicknesses ( $\mathrm{t}_{\text {Shell }}=0,2,3,3.5 \mathrm{~nm}$ ). (b) Dependence of the effective anisotropy, $\mathrm{K}_{\mathrm{Eff}}$, on the shell thickness, $\mathrm{t}_{\text {Shell. }}$ The symbols correspond to the experimental results, while the continuous line shows a $\mathrm{K}_{\mathrm{Eff}} \propto$ $1 /\left(R_{\text {core }}+t_{\text {Shell }}\right)^{3}$ dependence with $R_{\text {core }}=2.5 \mathrm{~nm}$. Shown in the inset is the dependence of the blocking temperature, $\mathrm{T}_{\mathrm{B}}$, on $\mathrm{t}_{\text {Shell. }}$. 


\section{Linear Absorption}

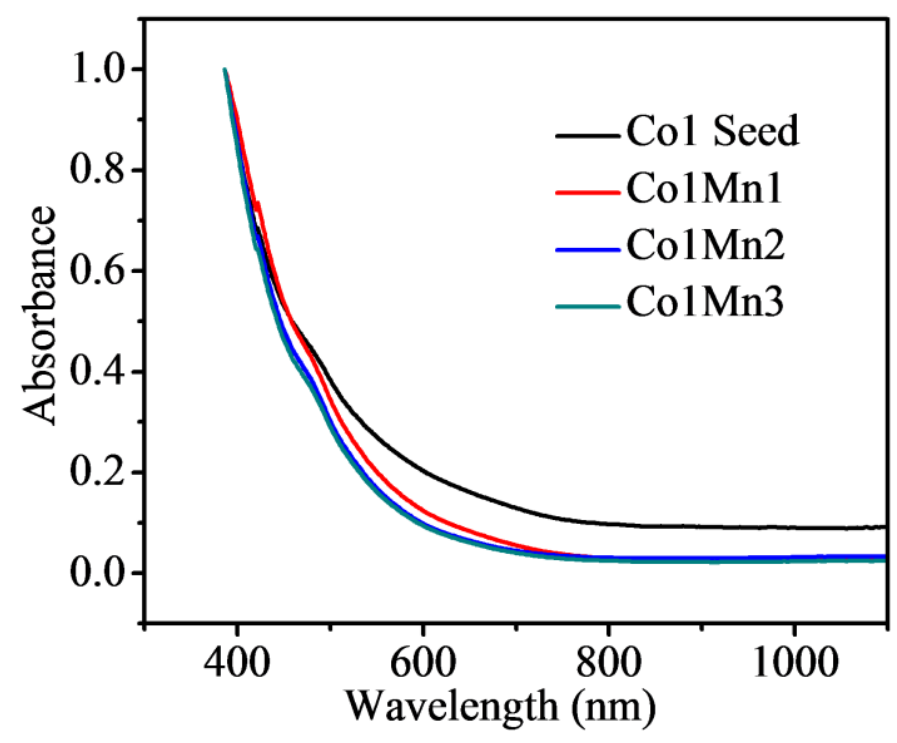

Figure S4. UV-Vis linear absorption spectra measured for the Co1@ series of nanoparticles.

\section{Z-scan Calibration Curve}

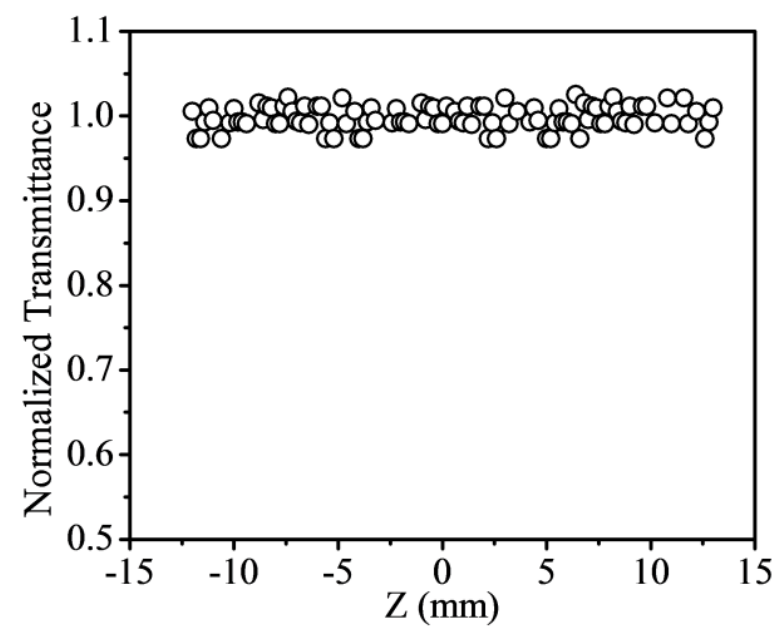

Figure S5. Open aperture Z-scan measured for the dispersing medium (pure toluene) used in the experiments (using $100 \mathrm{fs}, 800 \mathrm{~nm}, 10 \mu \mathrm{J}$ laser pulses). From the graph it is clear that toluene does not show any nonlinearity under these excitation conditions. 


\section{Z-scans for $800 \mathrm{~nm}-100 f$ pulses}

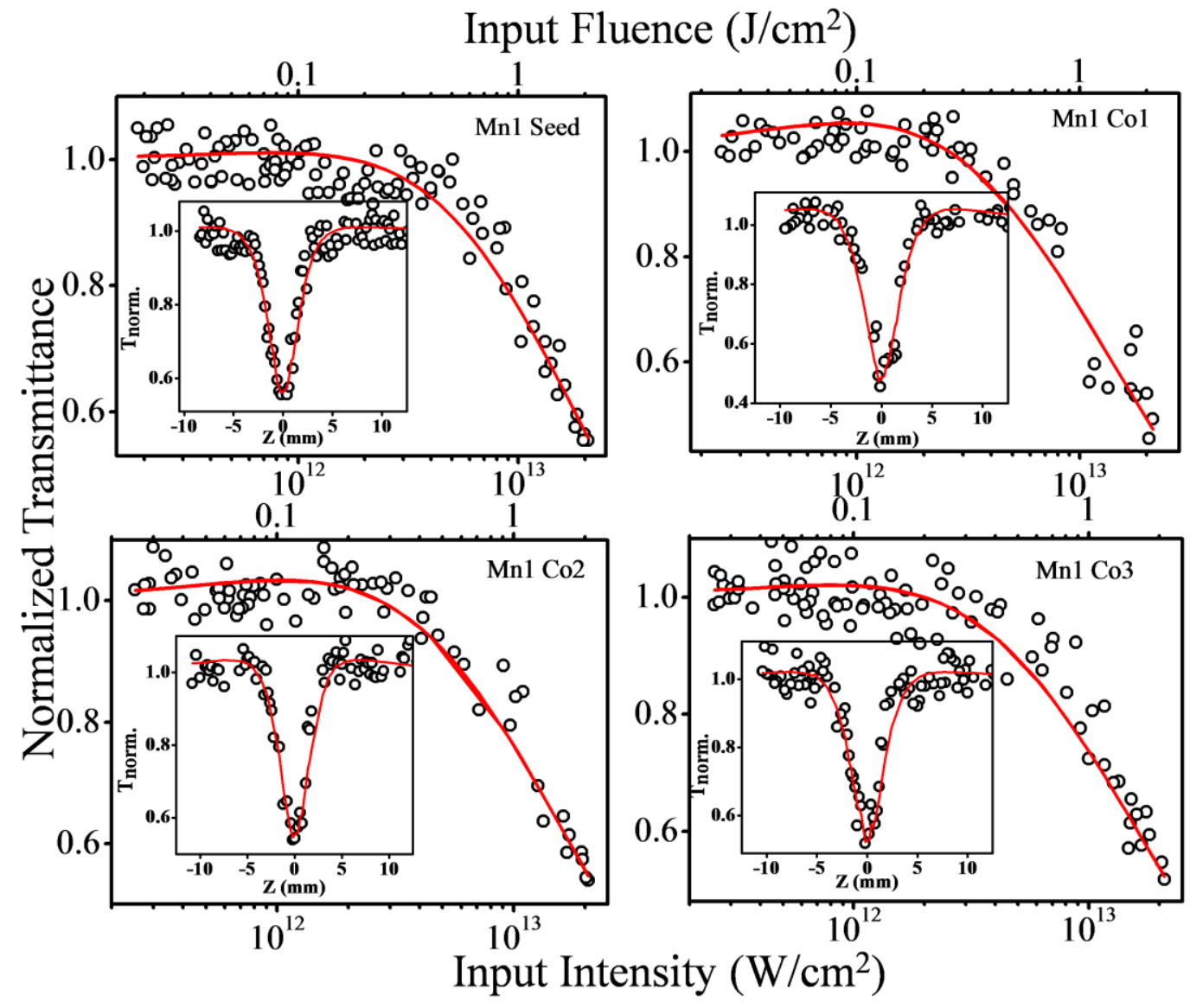

Figure S6. Normalized optical transmittance (for $800 \mathrm{~nm}, 10 \mu \mathrm{J}, 100 \mathrm{fs}$ pulses) obtained for the Mn1@Co series as a function of input intensity, calculated from the Z-scan curves (shown in the insets). The input fluence values also are shown. Symbols are experimental data, while the solid curves are numerical fits obtained using Equation (2), from which the $\beta$ and $I_{\mathrm{S}}$ values are determined. 


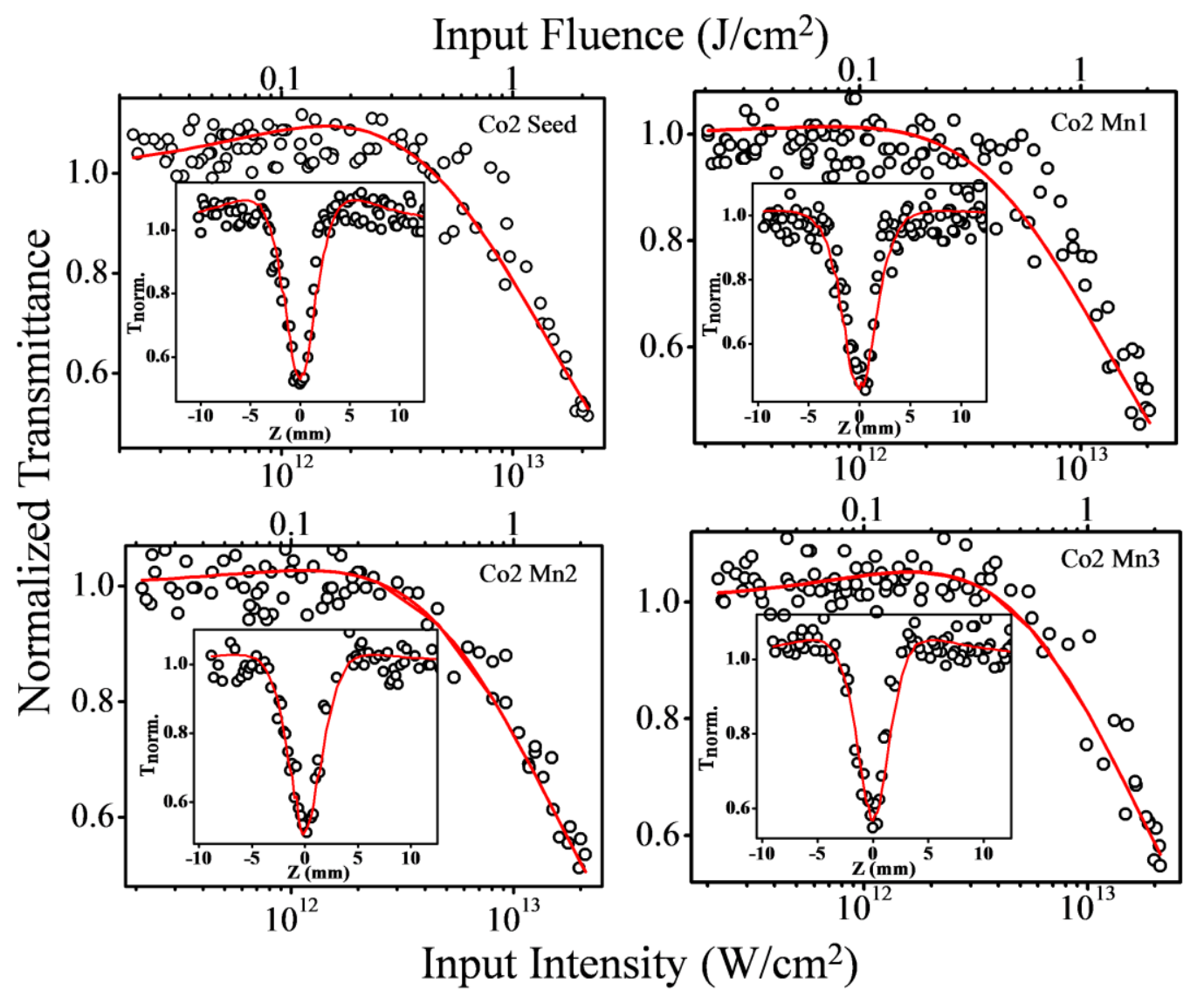

Figure S7. Normalized optical transmittance (for $800 \mathrm{~nm}, 10 \mu \mathrm{J}, 100 \mathrm{fs}$ pulses) obtained for the Co2@Mn series as a function of input intensity, calculated from the Z-scan curves (shown in the insets). The input fluence values also are shown. Symbols are experimental data, while the solid curves are numerical fits obtained using Equation (2), from which the $\beta$ and $I_{s}$ values are determined. 

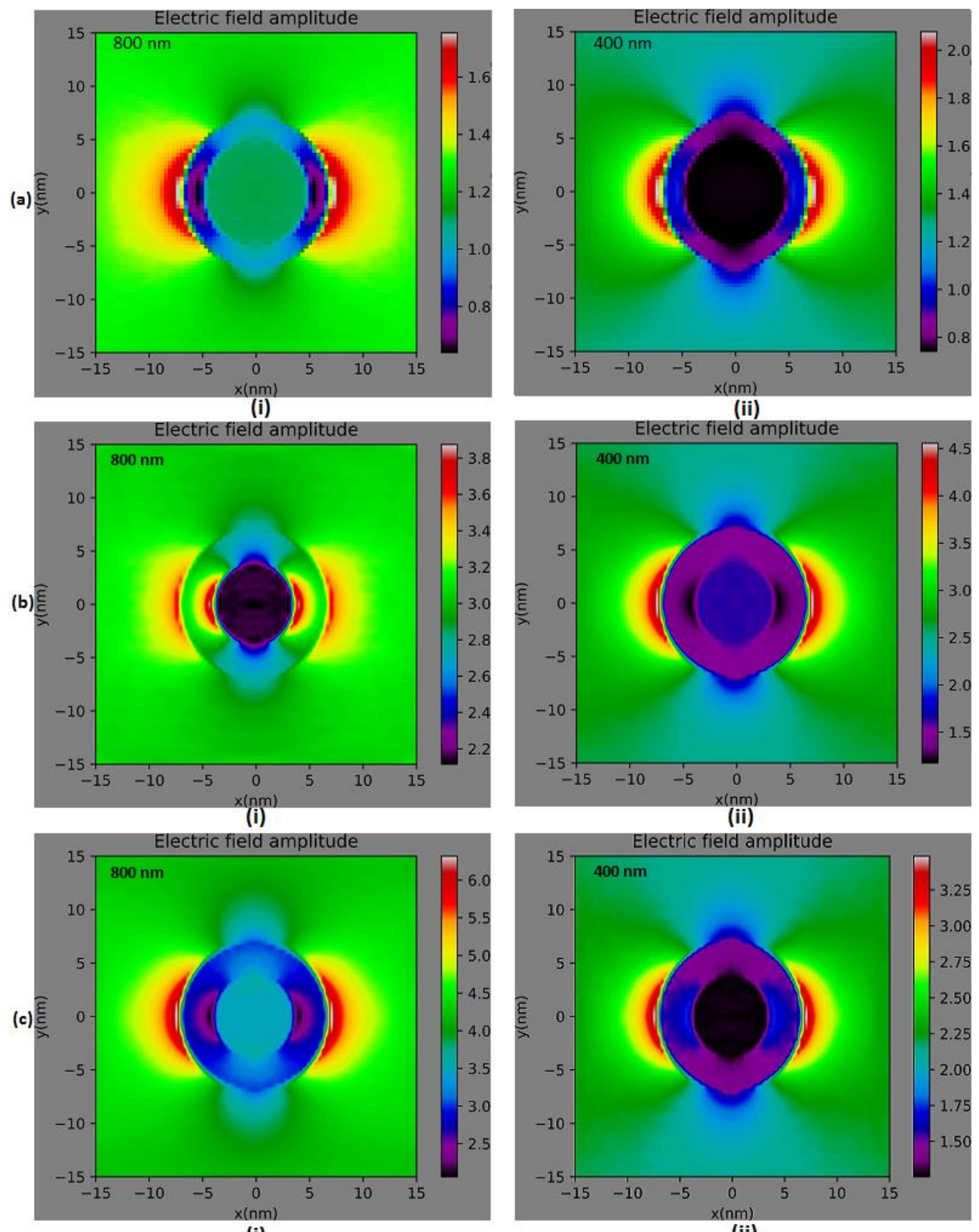

(i)

(ii)

Figure S8. FDTD simulations for (a) $\mathrm{CoFe}_{2} \mathrm{O}_{4} / \mathrm{MnFe}_{2} \mathrm{O}_{4}$ (core diameter $=8 \mathrm{~nm}$, shell thickness $=1.5 \mathrm{~nm}$ ), (b) $\mathrm{MnFe}_{2} \mathrm{O}_{4} / \mathrm{CoFe}_{2} \mathrm{O}_{4}$ (core diameter $=6 \mathrm{~nm}$, shell thickness $=2.5 \mathrm{~nm}$ ), and (c) $\mathrm{CoFe}_{2} \mathrm{O}_{4} / \mathrm{MnFe}_{2} \mathrm{O}_{4}$ (core diameter $=6 \mathrm{~nm}$, shell thickness $=2.5 \mathrm{~nm}$ ) for (i) $800 \mathrm{~nm}$ excitation, (ii) $400 \mathrm{~nm}$ excitation. The nanoparticles are periodically dispersed in the dielectric medium toluene, with an inter-particle separation of $30 \mathrm{~nm}$. The electric field amplitude within the nanoparticle and in its vicinity is plotted in a color scale, where the input field has been taken as unity. 


\section{Tables}

Table S1. Particle size, core diameter, shell thickness and crystallographic composition of core/shell Mn1@Co,Co1@Mn and Co2@Mn series.

\begin{tabular}{|c|c|c|c|c|c|c|}
\hline $\begin{array}{c}\text { Sample } \\
\text { code }\end{array}$ & $\begin{array}{c}\text { Total particle } \\
\text { size - TEM } \\
(\mathrm{nm})\end{array}$ & $\begin{array}{c}\text { Core } \\
\text { diameter } \\
(\mathrm{nm})\end{array}$ & $\begin{array}{c}\text { Shell } \\
\text { thickness } \\
(\mathrm{nm})\end{array}$ & $\begin{array}{c}\text { Cell } \\
\text { parameter } \\
(\mathrm{nm})\end{array}$ & $\begin{array}{l}\text { Crystallite } \\
\text { size - XRD } \\
(\mathrm{nm})\end{array}$ & $\begin{array}{c}\text { Crystallographic } \\
\text { Phase }\end{array}$ \\
\hline Mn1 seed & $6(1)$ & 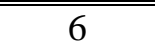 & 0 & " ------- & " ------- & $\mathrm{IMnFe}_{2} \mathrm{O}_{4}$ \\
\hline Mn1Co1 & $9(2)$ & 6 & 1.5 & ------- & ------- & $\mathrm{MnFe}_{2} \mathrm{O}_{4} / \mathrm{CoFe}_{2} \mathrm{O}_{4}$ \\
\hline Mn1Co2 & 11(1) & 6 & 2.5 & ------ & ------- & $\mathrm{MnFe}_{2} \mathrm{O}_{4} / \mathrm{CoFe}_{2} \mathrm{O}_{4}$ \\
\hline Mn1Co3 & $12(1)$ & 6 & 3 & ------- & ------ & $\mathrm{MnFe}_{2} \mathrm{O}_{4} / \mathrm{CoFe}_{2} \mathrm{O}_{4}$ \\
\hline Co1 seed & $6(1)$ & 6 & 0 & $0.8374(3)$ & $7(2)$ & $\mathrm{CoFe}_{2} \mathrm{O}_{4}$ \\
\hline Co1Mn1 & $9(1)$ & 6 & 1.5 & $0.8389(5)$ & 11(3) & $\mathrm{CoFe}_{2} \mathrm{O}_{4} / \mathrm{MnFe}_{2} \mathrm{O}_{4}$ \\
\hline Co1Mn2 & 11(1) & 6 & 2.5 & $0.8400(1)$ & $12(1)$ & $\mathrm{CoFe}_{2} \mathrm{O}_{4} / \mathrm{MnFe}_{2} \mathrm{O}_{4}$ \\
\hline Co1Mn3 & 12(1) & 6 & 3 & $0.8425(3)$ & 13(2) & $\mathrm{CoFe}_{2} \mathrm{O}_{4} / \mathrm{MnFe}_{2} \mathrm{O}_{4}$ \\
\hline Co2 seed & $8(1)$ & 8 & 0 & ------- & ------ & $\mathrm{CoFe}_{2} \mathrm{O}_{4}$ \\
\hline Co2Mn1 & $9(1)$ & 8 & 0.5 & ------- & ------- & $\mathrm{CoFe}_{2} \mathrm{O}_{4} / \mathrm{MnFe}_{2} \mathrm{O}_{4}$ \\
\hline Co2Mn2 & $11(2)$ & 8 & 1.5 & ------- & ------- & $\mathrm{CoFe}_{2} \mathrm{O}_{4} / \mathrm{MnFe}_{2} \mathrm{O}_{4}$ \\
\hline Co2Mn3 & $12(2)$ & 8 & 2 & ------- & ------- & $\mathrm{CoFe}_{2} \mathrm{O}_{4} / \mathrm{MnFe}_{2} \mathrm{O}_{4}$ \\
\hline
\end{tabular}

*Shown in brackets are the standard deviations of the Gaussian fit of the particle size distribution, see Figs. 1 and S1

Table S2. Effective two-photon absorption coefficients $(\beta)$ and saturation intensities $\left(\mathrm{I}_{\mathrm{s}}\right)$ obtained for $100 \mathrm{fs}$ pulse excitation at $800 \mathrm{~nm}$.

\begin{tabular}{cccc}
\hline \hline Sample & $\beta(\mathrm{cm} / \mathrm{W})$ & $\mathrm{I}_{\mathrm{s}}\left(\mathrm{W} / \mathrm{cm}^{2}\right)$ & $\mathrm{OL}^{*}$ threshold \\
\hline \hline Mn1 seed & $6.0 \times 10^{-13}$ & $2.8 \times 10^{12}$ & -- \\
Mn1Co1 & $8.0 \times 10^{-13}$ & $1.0 \times 10^{12}$ & 1.92 \\
Mn1Co2 & $6.4 \times 10^{-13}$ & $1.7 \times 10^{12}$ & -- \\
Mn1Co3 & $6.3 \times 10^{-13}$ & $1.7 \times 10^{12}$ & -- \\
\hline Co1 seed & $8.0 \times 10^{-13}$ & $1.0 \times 10^{12}$ & -- \\
Co1Mn1 & $1.2 \times 10^{-12}$ & $2.0 \times 10^{12}$ & 1.66 \\
Co1Mn2 & $1.1 \times 10^{-12}$ & $2.0 \times 10^{12}$ & 1.94 \\
Co1Mn3 & $1.0 \times 10^{-12}$ & $2.0 \times 10^{12}$ & 2.00 \\
\hline Co2 seed & $9.0 \times 10^{-13}$ & $1.7 \times 10^{12}$ & 2.04 \\
Co2Mn1 & $1.1 \times 10^{-12}$ & $3.0 \times 10^{12}$ & 1.75 \\
Co2Mn2 & $9.3 \times 10^{-13}$ & $3.0 \times 10^{12}$ & -- \\
Co2Mn3 & $7.8 \times 10^{-13}$ & $2.8 \times 10^{12}$ & -- \\
\hline \hline
\end{tabular}

${ }^{*}$ For some of the nanoparticles the OL threshold could not be accurately determined. 


\section{References}

[1] A. López-Ortega, M. Estrader, G. Salazar-Alvarez, A.G. Roca, J. Nogués, Phys. Rep. $\mathbf{2 0 1 5}, 553,1-32$. 